\title{
Seismological investigations in the Gioia Tauro Basin (southern Calabria, Italy)
}

\author{
Elisabetta Giampiccolo $\left({ }^{1}\right)$, Carla Musumeci $\left({ }^{1}\right)$, Filippo Falà $\left({ }^{2}\right)$ and Stefano Gresta $\left({ }^{2}\right)$ \\ (') Istituto Nazionale di Geofisica e Vulcanologia, Sezione di Catania, Catania, Italy \\ ( $\left.{ }^{2}\right)$ Dipartimento di Scienze Geologiche, Università degli Studi di Catania, Italy
}

\begin{abstract}
This study provides new seismological information to characterize the seismically active area of the Gioia Tauro basin (southern Calabria, Italy). Seismic activity recorded by a temporary network from 1985 to 1994 was analyzed for focal mechanisms, stress tensor inversion, P-wave seismic attenuation and earthquake source parameters estimation. Fault plane solutions of selected events showed a variety of different mechanisms, even if a prevalence of normal dip-slip solutions with prevalent rupture orientations occurring along ca. NE-SW directions was observed. Stress tensor inversion analysis disclosed a region governed mainly by a NW-SE extensional stress regime with a nearly vertical $\sigma_{1}$. These results are consistent with the structure movements affecting the studied area and with geodetic data.

Furthermore, evaluation of P-waves seismic attenuation and earthquake source parameters of a subset of events highlighted a strong heterogeneity of the crust and the presence of fault segments and/or weakened zones where great stress accumulation or long-rupture propagation are hindered.
\end{abstract}

Key words Southern Calabria-Seismicity - Stress tensor - Attenuation - Source parameters

\section{Introduction}

The Calabrian arc (fig. 1) is the most arcuate southern part of the Mediterranean orogenic belt. The arc connects the E-W and the NW-SE trending branches of the belt, which are represented by the Maghrebian and the southern Apennines chains, respectively. The most impressive tectonic feature of the arc is a prominent normal fault belt that extends, more or less continuously, for a total length of about $180 \mathrm{~km}$ along the inner side of the arc (Tortorici et al., 1995). The morphological features of the fault

Mailing address: Dr. Elisabetta Giampiccolo, Istituto Nazionale di Geofisica e Vulcanologia, Sezione di Catania, Piazza Roma 2, 95123 Catania, Italy; e-mail: giampiccolo@ct.ingv.it escarpments suggest slip rates of $0.8-1.1 \mathrm{~mm} / \mathrm{yr}$ for the last $700 \mathrm{k} . y$. and values of $0.6-0.9 \mathrm{~mm} /$ $\mathrm{yr}$ for the last $120 \mathrm{k.y}$., indicating a uniform rate of faulting since the Middle Pleistocene (Tortorici et al., 1995). The different normal fault segments separate the main Pliocene-Pleistocene basins from the uplifted mountain ranges (Tortorici et al., 1995). The study area (fig. 1) is a ca. $40 \mathrm{~km}$ long section of the Calabrian arc that includes the Gioia-Tauro basin and the Aspromonte mountain range. In particular, the Gioia Tauro basin (fig. 1) is part of a system of basins that border the Serre-Aspromonte toward the western coast of southern Italy. It is characterized by the Cittanova fault (hereafter referred as $\mathrm{CF}$ ), a well exposed west dipping high-angle normal fault about $20 \mathrm{~km}$ long, which separates metamorphic and igneous rocks to the east from sedimentary marine and continental successions to the west (Galli and Bosi, 2002). In the field, the CF appears both as a single $\sim 10 \mathrm{~m}$ high scarp or as smaller multiple 


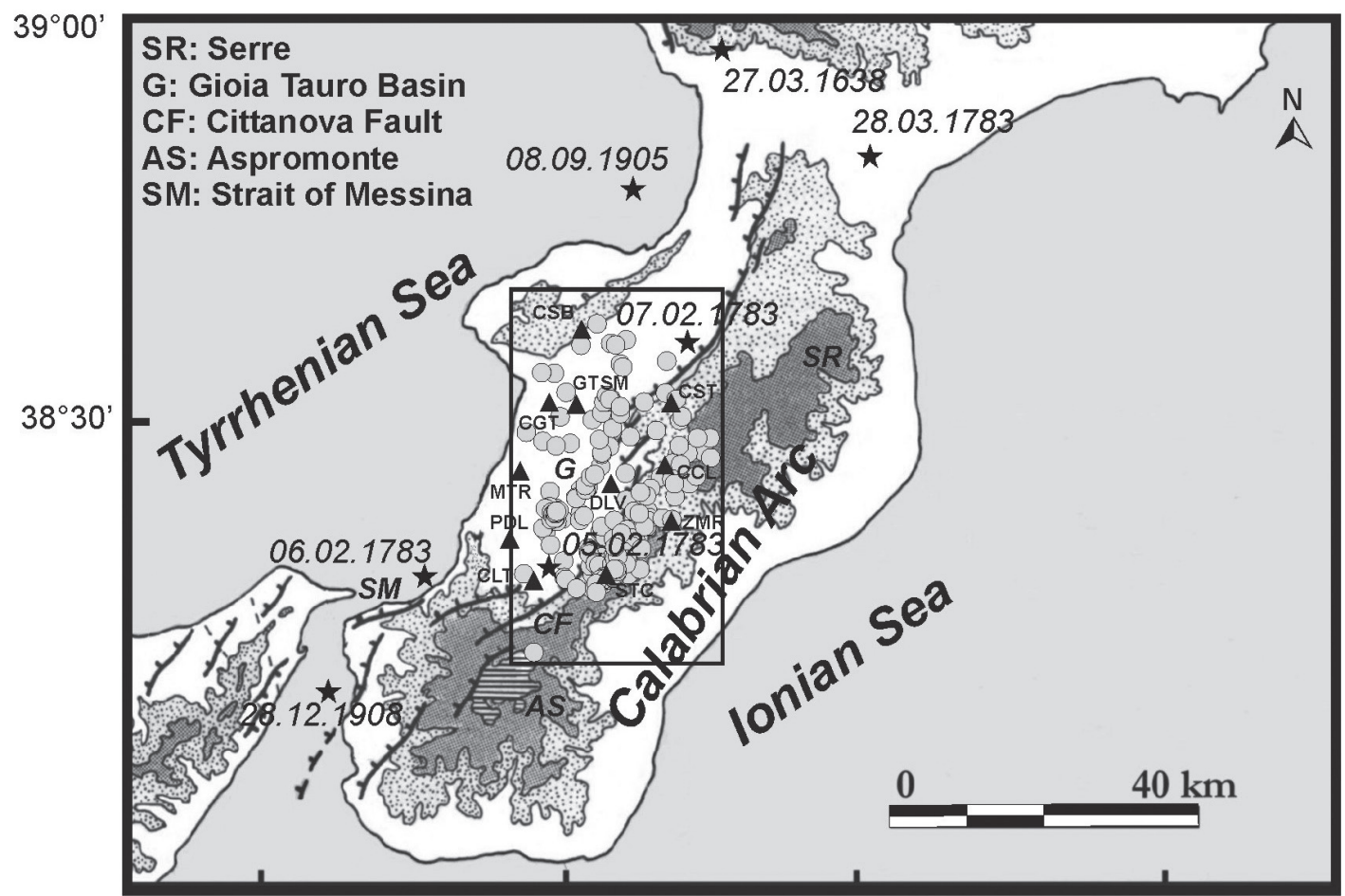

$15^{\circ} 30^{\prime}$

$17^{\circ} 00^{\prime}$

Fig. 1. Map of the study area (modified after Tortorici et al., 1995) showing the location of the events selected in this study (grey circles inside the black rectangle). Historical earthquakes are also shown (stars). Triangles represent the stations of the seismic network operating from 1985 to 1994.

scarpets, with a 1 to $3 \mathrm{~m}$ high frontal scarp that, in some places, has a free face (Wallace, 1977). It is composed of a dozen en echelon strands, 1-3 km long which strike $\mathrm{N} 40^{\circ} \mathrm{E}$, with the exception of two $\mathrm{N} 25^{\circ} \mathrm{E}$ relay ramps. Almost all the strands show a right step with respect to the northern ones (Galli and Bosi, 2002).

From a seismic point of view, the Calabrian arc represents one of the most active zones in Europe. It has experienced the strongest earthquakes affecting the Italian region over the last 3 centuries (fig. 1): 1638 (Io=IX MCS), 1783 (Io=X-XI MCS), 1905 (Io=X-XI MCS), 1908 (Io=XI MCS) (e.g. Boschi et al., 2000; Monaco and Tortorici, 2000; Galli and Bosi, 2003). The strongest events (except those of 1638) occurred in the southern sector, between the Messina and Catanzaro straits. In particular, the
1783 earthquakes were a catastrophic and destructive sequence for an area more than 100 $\mathrm{km}$ in length and $30 \mathrm{~km}$ in width (Tyrrhenian coast and front of the Aspromonte-Serre Range). The CF is generally identified as the superficial expression of the seismogenetic structure of the February 5, 1783 earthquake (Galli and Bosi, 2002). However, the lack of geological evidence concerning middle-upper Pleistocene activity of the CF has led some authors to question its present activity and to search for another seismic source elsewhere. Valensise and D'Addezio (1994), among others, claim that the $\mathrm{CF}$ is the fossil trace of an inactive fault, or an ancient coast line. The authors assume that the fault controlling the geometry and the seismicity of the Gioia Tauro basin is instead a blind, low-angle, east dipping fault system (Gioia 
Tauro Fault). According to recent paleoseismological and archeoseismological studies (e.g. Galli and Bosi, 2002) the CF is still active and has produced several significant events in the Holocene and historical times.

The occurrence in the Calabrian arc of both intense Quaternary faulting and active crustal seismicity suggests that these phenomena might be related to each other. Therefore, with the aim of monitoring the seismic activity of the Gioia Tauro basin, a local seismic network was managed, on behalf of ENEL (Italian national electricity board), from April 1985 to April 1994 by ISMES (Istituto Sperimentale Modelli E Strutture), which provided for a catalogue of the recorded seismicity too. Despite the large amount of recorded local earthquakes over a 9-year time span, little effort has been made so far to carry out detailed researches on seismological topics. Recently, Raffaele et al. (2006) investigated the three-dimensional velocity structure of the Gioia Tauro basin providing important constraints on the structural and geological features. However, more studies and researches are needed to better understand and explain both tectonics and physical mechanisms occurring in this seismically active area. As is known, the assessment of seismic hazard requires information on both the nature of the earthquake source and on the medium where seismic waves propagate. In this framework, we attempt a systematic and detailed analysis of a 9-year time span, which includes the most complete and largest dataset available to date in southern Calabria. Our goals are: i) to provide a dataset of well-constrained fault plane solutions and source parameters for a selected subset of earthquakes; ii) to give information on the distribution of stress direction and discuss the results in terms of spatial earthquake distribution and tectonics; iii) to obtain a first estimate of the seismic attenuation of P-waves which may provide important insights into the nature of heterogeneities in the study area.

\section{Instruments and Data}

A seismic network of 11 stations was installed in the Gioia Tauro basin between April
1985 and April 1994. Until July 1992 the network was equipped with one three component (DLV) and ten vertical component stations. After July 1992 also PDL and CST were run as three component seismic stations (Raffaele et al., 2006). Each station was equipped with Mark L4C and Mark L4C-3D seismometers (flat response between 0.8 and $30 \mathrm{~Hz}$; Moia, 1987) having a natural frequency of $1 \mathrm{~Hz}$ and a damping of $70 \%$ of critical. The data were sampled with a sampling rate of $153.8 \mathrm{~Hz}$.

Among 3741 events recorded during the 9 years of the network's operation, we selected a subset of 252 events from the ISMES catalogue within an area defined by a rectangle with coordinates 38.16-38.65 latitude $\mathrm{N}^{\circ}$ and 15.8916.30 longitude $\mathrm{E}^{\circ}$ (fig. 1). The quality of the input data was improved by repicking the Pand S-phases. The earthquakes were then relocated (table I) using the Hypoellipse code (Lahr, 1989) and the minimum 1D velocity model proposed by Raffaele et al. (2006). Final locations are affected by uncertainty less than 2 $\mathrm{km}$ in epicentral coordinates $(80 \%$ Erh $\leq 1.0$ $\mathrm{km})$, less than $3 \mathrm{~km}$ in focal depths $(50 \%$ Erz $\leq 1.0 \mathrm{~km}$ ), and root-mean-square (rms) traveltime residual less than $0.25 \mathrm{~s}$. Higher depth errors are mainly observed for the events occurring along the western coastal sector of the study area because of the largest azimuthal gaps. Since high-precision hypocentral coordinates and reliable error-estimates are crucial for seismo-tectonic interpretation, we used the differences between minimum 1D and 3D model locations (Raffaele et al., 2006) to verify the accuracy of hypocentral coordinates. These location differences are rather small with an average location accuracy of about $0.3 \mathrm{~km}$ in horizontal directions and about $0.8 \mathrm{~km}$ in depth for the selected well locatable events.

The spatial distribution of seismicity shows an Appenninic trend along a NE-SW direction (fig. 1). The hypocentral distribution of the events extends to about $25 \mathrm{~km}$, with depth mainly concentrated from 10 to $25 \mathrm{~km}$. The local magnitude $\left(M_{L}\right)$ of the events was calculated following the procedure implemented in the Hypoellipse code (Lahr, 1989) which proved to give very similar magnitude values to those calculated following the standard Richter 
Table I. Location parameters of the 252 events selected from the ISMES catalogue, including local magnitude $\left(\mathrm{M}_{\mathrm{L}}\right)$, number of data $(\mathrm{N})$, azimuthal gap (Gap), squared residual travel times (rms), Erh and Erz.

\begin{tabular}{|c|c|c|c|c|c|c|c|c|c|c|c|}
\hline $\mathbf{N}^{\circ}$ & Date & Orig. Time & $\mathbf{M}_{\mathbf{L}}$ & Lat. $\mathbf{N}^{\circ}$ & Long. $\mathrm{E}^{\circ}$ & Depth & $\mathbf{N}$ & Gap & rms & Erh & Erz \\
\hline 1 & $14 / 04 / 1985$ & 03:31:22.04 & 0.5 & 38.310 & 16.038 & 18.75 & 8 & 119 & 0.06 & 0.8 & 1 \\
\hline 2 & $28 / 04 / 1985$ & $22: 01: 49.64$ & 0.5 & 38.376 & 15.994 & 13.5 & 7 & 127 & 0.08 & 0.7 & 0.7 \\
\hline 3 & $28 / 04 / 1985$ & $22: 19: 51.32$ & 0.7 & 38.378 & 15.995 & 13.75 & 7 & 99 & 0.06 & 0.7 & 0.7 \\
\hline 4 & $28 / 04 / 1985$ & $22: 41: 03.14$ & 1.4 & 38.374 & 15.991 & 13.43 & 10 & 128 & 0.07 & 0.5 & 0.7 \\
\hline 5 & $20 / 05 / 1985$ & 01:45:07.67 & 0.5 & 38.372 & 15.931 & 20.27 & 8 & 200 & 0.04 & 1 & 0.8 \\
\hline 6 & $27 / 06 / 1985$ & $22: 27: 58.56$ & 2.0 & 38.405 & 16.016 & 58.13 & 9 & 89 & 0.09 & 1.8 & 2.1 \\
\hline 7 & $19 / 07 / 1985$ & 07:56:06.96 & 0.5 & 38.281 & 15.954 & 10.28 & 6 & 184 & 0.05 & 1.7 & 2.6 \\
\hline 8 & $24 / 08 / 1985$ & $17: 56: 37.65$ & 1.0 & 38.319 & 16.019 & 12.59 & 8 & 132 & 0.07 & 1 & 1.9 \\
\hline 9 & $31 / 08 / 1985$ & $21: 22: 08.53$ & 0.5 & 38.470 & 15.948 & 15.09 & 6 & 106 & 0.06 & 0.7 & 2.1 \\
\hline 10 & $26 / 10 / 1985$ & $16: 52: 03.04$ & 1.2 & 38.279 & 16.008 & 17.47 & 8 & 117 & 0.05 & 0.9 & 1.3 \\
\hline 11 & $27 / 10 / 1985$ & $06: 44: 54.88$ & 1.5 & 38.277 & 16.007 & 17.22 & 7 & 120 & 0.05 & 1 & 1.4 \\
\hline 12 & $28 / 10 / 1985$ & $18: 41: 47.79$ & 2.2 & 38.294 & 16.047 & 18.88 & 11 & 149 & 0.07 & 1 & 1.3 \\
\hline 13 & $13 / 11 / 1985$ & $13: 29: 46.27$ & 0.5 & 38.455 & 16.036 & 0.74 & 6 & 15 & 0.14 & 0.7 & 3 \\
\hline 14 & $20 / 11 / 1985$ & $17: 07: 22.24$ & 1.7 & 38.288 & 16.038 & 18.49 & 9 & 14 & 0.05 & 0.7 & 0.8 \\
\hline 15 & $11 / 12 / 1985$ & $22: 11: 23.52$ & 1.2 & 38.571 & 16.059 & 22.77 & 8 & 222 & 0.04 & 1 & 1 \\
\hline 16 & $26 / 12 / 1985$ & $00: 46: 51.07$ & 0.6 & 38.361 & 16.059 & 20.63 & 8 & 80 & 0.04 & 0.8 & 0.8 \\
\hline 17 & $27 / 12 / 1985$ & $09: 56: 21.52$ & 1.2 & 38.258 & 16.025 & 19.64 & 6 & 272 & 0.02 & 1.1 & 1.3 \\
\hline 18 & $27 / 12 / 1985$ & 18:00:10.63 & 2.0 & 38.263 & 16.024 & 19.33 & 11 & 214 & 0.06 & 0.7 & 0.8 \\
\hline 19 & $27 / 12 / 1985$ & $20: 05: 48.43$ & 0.6 & & 16.02 & 19.16 & 6 & 245 & & 1.1 & 1.4 \\
\hline 20 & $27 / 12 / 1985$ & 20:07:31.85 & 1.2 & 38.264 & 16.02 & 19.33 & 9 & 176 & 0 & 0.7 & 0.9 \\
\hline 21 & $27 / 12 / 1985$ & 20:07:49.31 & 1.4 & 38.261 & 16.032 & 18.27 & 10 & 219 & 0.12 & 0.7 & 0.8 \\
\hline 22 & $28 / 12 / 1985$ & $14: 52: 24.25$ & 1.9 & 38.264 & 16.026 & 19.79 & 10 & 214 & 0.06 & 0.7 & 0.8 \\
\hline 23 & $29 / 12 / 1985$ & 19:56:02.99 & 1.0 & 38.258 & 16.022 & 18.8 & 7 & 243 & 0.01 & 0.9 & 1.1 \\
\hline 24 & 04/01/1986 & 02:37:06.54 & 1.6 & 38.262 & 16.023 & 19.02 & 11 & 215 & 0.06 & 0.7 & 0.8 \\
\hline 25 & $30 / 01 / 1986$ & $06: 22: 10.86$ & 1.5 & 38.307 & 16.075 & 20.12 & 7 & 187 & 0.1 & 1.2 & 1.2 \\
\hline 26 & $04 / 02 / 1986$ & $22: 44: 05.52$ & 0.7 & 38.322 & 16.06 & 16.56 & 6 & 125 & 0.04 & 1.3 & 2.2 \\
\hline 27 & 05/02/1986 & 00:00:30.34 & 0.8 & 38.311 & 16.067 & 19.63 & 8 & 148 & 0.05 & 0.8 & 0.8 \\
\hline 28 & $23 / 02 / 1986$ & $15: 33: 29.42$ & 1.5 & 38.260 & 16.022 & 19.21 & 8 & 221 & 0.02 & 0.8 & 0.9 \\
\hline 29 & $23 / 02 / 1986$ & $15: 33: 47.76$ & 1.5 & 38.263 & 16.031 & 18.08 & 9 & 216 & 0.1 & 0.8 & 1.1 \\
\hline 30 & $23 / 02 / 1986$ & $15: 42: 31.76$ & 1.2 & 38.260 & 16.029 & 18.23 & 9 & 218 & 0.1 & 0.8 & 1 \\
\hline 31 & $24 / 02 / 1986$ & 10:09:56.51 & 1.5 & 38.266 & 16.017 & 18.78 & 10 & 150 & 0.1 & 0.6 & 0.8 \\
\hline 32 & 08/03/1986 & 03:11:28.64 & 1.3 & 38.263 & 16.029 & 18.1 & 12 & 216 & 0.09 & 0.7 & 0.7 \\
\hline 33 & 08/03/1986 & 17:39:09.65 & 1.0 & 38.263 & 16.028 & 19.1 & 9 & 215 & 0.06 & 0.7 & 0.8 \\
\hline 34 & $12 / 03 / 1986$ & 03:34:09.64 & 2.4 & 38.260 & 16.022 & 18.66 & 11 & 206 & 0.08 & 0.7 & 0.8 \\
\hline 35 & $12 / 03 / 1986$ & $03: 34: 13.93$ & 2.2 & 38.261 & 16.026 & 18.66 & 11 & 206 & 0.08 & 0.7 & 0.8 \\
\hline
\end{tabular}


Table I. (continued).

\begin{tabular}{|c|c|c|c|c|c|c|c|c|c|c|c|}
\hline $\mathbf{N}^{\circ}$ & Date & Orig. Time & $\mathbf{M}_{\mathbf{L}}$ & Lat. $\mathrm{N}^{\circ}$ & Long. $\mathrm{E}^{\circ}$ & Depth & $\mathbf{N}$ & Gap & rms & Erh & Erz \\
\hline 36 & $12 / 03 / 1986$ & $03: 36: 04.43$ & 2.2 & 38.262 & 16.027 & 18.64 & 11 & 204 & 0.07 & 0.7 & 0.8 \\
\hline 37 & $12 / 03 / 1986$ & 03:39:14.88 & 1.1 & 38.256 & 16.026 & 19.29 & $\pi_{0}$ & 222 & 0.04 & .9 & 1.4 \\
\hline 38 & $12 / 03 / 1986$ & 03:54:00.19 & 1.9 & 38.259 & 16.022 & 18.87 & 11 & 217 & 0.06 & .7 & 0.8 \\
\hline 39 & $12 / 03 / 1986$ & 05:00:06.66 & 1.0 & 38.250 & 16.02 & 17.29 & 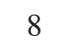 & 248 & 0.06 & 0.8 & 1 \\
\hline 40 & $12 / 03 / 1986$ & $05: 49: 58.83$ & 0.9 & 38.257 & 16.022 & 18.89 & 6 & 244 & 0.02 & 1 & 1.5 \\
\hline 41 & $12 / 03 / 1986$ & $05: 53: 25.81$ & 2.1 & 38.260 & 16.021 & 18.66 & 11 & 216 & 0.07 & 0.7 & 0.8 \\
\hline 42 & $12 / 03 / 1986$ & $06: 58: 59.54$ & 1.7 & 38.257 & 16.017 & 18.61 & 9 & 213 & 0.07 & 0.7 & 0.8 \\
\hline 43 & $12 / 03 / 1986$ & $09: 27: 21.56$ & 1.5 & 38.265 & 16.026 & 19.28 & 9 & 170 & 0.06 & 0.7 & 0.8 \\
\hline 44 & $12 / 03 / 1986$ & $15: 21: 38.95$ & 1.4 & 38.261 & 16.022 & 19.11 & 9 & 216 & 0.06 & 0.7 & 0.9 \\
\hline 45 & 12/03/1986 & $18: 55: 09.54$ & 1.5 & 38.263 & 16.023 & 18.94 & 10 & 214 & 0.06 & .7 & 0.8 \\
\hline 46 & 21/03/1986 & $06: 01: 18.65$ & 1.9 & 38.261 & 16.02 & .28 & 10 & 215 & 0.06 & .7 & 0.8 \\
\hline 47 & 01/04/1986 & $22: 04: 28.45$ & 1.1 & & 16.149 & & 9 & 201 & 0.03 & .8 & 0.9 \\
\hline 48 & 13/04/1986 & $03: 56: 22.62$ & 1.0 & & & & 6 & 162 & 0.01 & .5 & 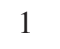 \\
\hline 49 & 17/06/1986 & $18: 46: 00.44$ & 2.0 & 38.566 & 16.033 & 21.49 & 10 & 199 & & .7 & 1 \\
\hline 50 & 17/07/1986 & $07: 3$ & 1.2 & 38.257 & 16 & 18.72 & . & 244 & 0.02 & 9 & 1.1 \\
\hline 51 & 29/07/1986 & 19:19:28.52 & 1.0 & 38.319 & 15.924 & 21.2 & , & 77 & 0.06 & .7 & 0.9 \\
\hline 52 & 02/08/1986 & $11: 20: 52.40$ & 0.9 & 38.324 & 15.92 & 22.01 & 6 & 127 & 0.04 & .1 & .1 \\
\hline 53 & 02/08/1986 & $12: 27: 39.72$ & 0.9 & 38.324 & 15.919 & 21.85 & 6 & 164 & 0.03 & 1.5 & 1.2 \\
\hline 54 & 08/08/1986 & $19: 30: 52.80$ & 0.6 & 38.284 & 16.04 & 19.47 & 6 & 155 & 0.02 & 0.9 & 1.1 \\
\hline 55 & 24/08/1986 & $02: 38: 37.50$ & 1.1 & 38.286 & 16.018 & 12.81 & 9 & 104 & 0.07 & 0.6 & 0.9 \\
\hline 56 & 24/08/1986 & 02:52:31.69 & 1.1 & 38.257 & 16.023 & 18.27 & 8 & 244 & 0.02 & 0.8 & 0.9 \\
\hline 57 & 24/08/1986 & $20: 13: 25.54$ & 1.5 & 38.273 & 16.022 & 16.78 & 8 & 110 & 0.06 & 0.7 & 1.1 \\
\hline 58 & 26/08/1986 & $06: 54: 18.47$ & 1.6 & 38.263 & 16.028 & 19.54 & 8 & 215 & 0.07 & 0.8 & 1.1 \\
\hline 59 & 26/08/1986 & $14: 15: 29.71$ & 1.1 & 38.265 & 16.027 & 18.9 & 8 & 177 & 0.07 & 0.8 & 1.1 \\
\hline 60 & 23/09/1986 & $20: 20: 35.27$ & 1.1 & 38.365 & 16.141 & 15.37 & 8 & 191 & 0.06 & 0.7 & 0.8 \\
\hline 61 & 19/10/1986 & 04:09:52.22 & 1.3 & 38.264 & 16.017 & & 7 & 172 & 0.08 & 0.9 & 1.1 \\
\hline 62 & 19/10/1986 & $23: 38: 14.83$ & 1.0 & & & & 6 & 214 & 0.05 & 1.5 & 1.8 \\
\hline 63 & 20/10/1986 & $13: 17: 04.23$ & 1.2 & 38.380 & 15.99 & 12.83 & 8 & 123 & 0.12 & 0.8 & 0.7 \\
\hline 64 & 27/11/1986 & $19: 26: 51.38$ & 1.1 & 38.251 & 16.009 & 21.16 & 8 & 223 & 0.06 & 0.9 & 1.2 \\
\hline 65 & $13 / 12 / 1986$ & $00: 25: 35.03$ & 1.2 & 38.336 & 15.952 & 20.72 & 10 & 114 & 0.08 & 0.7 & 0.8 \\
\hline 66 & $14 / 12 / 1986$ & 01:08:56.81 & 0.7 & 38.391 & 16.117 & 9.58 & 6 & 148 & 0.02 & 0.9 & 1.9 \\
\hline 67 & $16 / 12 / 1986$ & 02:13:16.01 & 1.7 & 38.335 & 16.136 & 19.73 & 10 & 206 & 0.07 & 0.7 & 0.8 \\
\hline 68 & 19/03/1987 & 20:23:05.35 & 0.7 & 38.424 & 16.189 & 9.96 & 7 & 238 & 0.09 & 0.8 & 1.3 \\
\hline 69 & 14/04/1987 & $13: 49: 22.08$ & 0.5 & 38.343 & 15.982 & 14.28 & 6 & 148 & 0.1 & 1.4 & 3 \\
\hline 70 & $14 / 04 / 1987$ & $13: 53: 47.53$ & 1.4 & 38.334 & 15.981 & 21.28 & 9 & 101 & 0.04 & 0.9 & 1.1 \\
\hline 71 & $16 / 04 / 1987$ & 09:12:42.77 & 1.1 & 38.340 & 15.988 & 19.31 & 6 & 146 & 0.02 & 1.2 & 1.6 \\
\hline
\end{tabular}


Table I. (continued).

\begin{tabular}{|c|c|c|c|c|c|c|c|c|c|c|c|}
\hline $\mathbf{N}^{\circ}$ & Date & Orig. Time & $\mathbf{M}_{\mathbf{L}}$ & Lat. $\mathbf{N}^{\circ}$ & Long. $\mathrm{E}^{\circ}$ & Depth & $\mathbf{N}$ & Gap & rms & Erh & Erz \\
\hline 72 & 03/05/1987 & 04:07:40.12 & 1.7 & 38.592 & 16.01 & 20.94 & 9 & 235 & 0.08 & 0.8 & 1.1 \\
\hline 73 & $12 / 05 / 1987$ & $11: 34: 11.23$ & 1.2 & 38.423 & 16.152 & 18.96 & 6 & 285 & 0 & 1.3 & 0.9 \\
\hline 74 & $12 / 05 / 1987$ & $11: 36: 06.70$ & 1.4 & 38.420 & 16.145 & 18.97 & 7 & 281 & 0.04 & 1.3 & 0.8 \\
\hline 75 & $12 / 05 / 1987$ & $12: 11: 15.13$ & 1.1 & 38.409 & 16.142 & 18.46 & 8 & 220 & 0.05 & 0.8 & 0.8 \\
\hline 76 & $12 / 05 / 1987$ & 12:11:59.94 & 1.4 & 38.407 & 16.147 & 19.3 & 6 & 194 & 0.04 & 1.2 & 1.3 \\
\hline 77 & $12 / 05 / 1987$ & $12: 14: 59.85$ & 1.7 & 38.410 & 16.144 & 18.28 & 10 & 191 & 0.07 & 0.7 & 0.7 \\
\hline 78 & $12 / 05 / 1987$ & $12: 17: 39.33$ & 1.5 & 38.404 & 16.141 & 17.67 & 11 & 188 & 0.09 & 0.7 & 0.7 \\
\hline 79 & $12 / 05 / 1987$ & $12: 27: 26.53$ & 1.2 & 38.427 & 16.15 & 18.46 & 6 & 285 & 0.02 & 1.6 & 0.9 \\
\hline 80 & $12 / 05 / 1987$ & $12: 28: 38.11$ & 2.0 & 38.409 & 16.137 & 17.7 & 11 & 183 & 0.06 & 0.7 & 0.7 \\
\hline 81 & $12 / 05 / 1987$ & $14: 58: 19.51$ & 1.1 & 38.422 & 16.163 & 17.07 & 9 & 213 & 0.12 & 0.8 & 0.8 \\
\hline 82 & $16 / 05 / 1987$ & 01:14:47.55 & 1.3 & 38.489 & 16.089 & 21.78 & 8 & 132 & 0.05 & 0.8 & 0.9 \\
\hline 83 & $16 / 05 / 1987$ & 10:59:31.36 & 1.3 & 38.407 & 16.15 & & 9 & & 0.05 & 0.7 & 0.9 \\
\hline 84 & $14 / 06 / 1987$ & & 2.0 & & & & 10 & & 0.08 & 1 & 0.8 \\
\hline 85 & 28/07/1987 & & 1.4 & & 15.938 & & 9 & & 0.1 & 0.7 & 1.1 \\
\hline 86 & $28 / 07 / 1987$ & 20:05:59.39 & 0.9 & & 15.917 & & 6 & & 0.04 & 1 & 1.7 \\
\hline 87 & $19 / 08 / 1987$ & $23: 21: 19.28$ & 0.5 & 38.300 & 16.07 & 19.45 & 6 & 203 & 0.02 & 1 & 0.9 \\
\hline 88 & $20 / 10 / 1987$ & 20:06:39.73 & 1.2 & 38.474 & 16.049 & 20.8 & 7 & 159 & 0.01 & 1 & 1.1 \\
\hline 89 & $18 / 12 / 1987$ & $21: 17: 29.50$ & 1.3 & 38.259 & 15.955 & 22.24 & 7 & 180 & 0.04 & 1.5 & 1.2 \\
\hline 90 & $24 / 01 / 1988$ & 01:50:50.12 & 3.0 & 38.350 & 15.941 & 18.55 & 8 & 79 & 0.05 & 0.7 & 2.5 \\
\hline 91 & $24 / 01 / 1988$ & $18: 45: 36.87$ & 1.7 & 38.348 & 15.936 & 20.67 & 8 & 83 & 0.06 & 0.8 & 0.8 \\
\hline 92 & $25 / 01 / 1988$ & $16: 24: 37.82$ & 1.7 & 38.352 & 15.93 & 21.29 & 7 & 89 & 0.03 & 0.9 & 0.9 \\
\hline 93 & 27/01/1988 & $07: 35: 41.80$ & 1.6 & 38.350 & 15.923 & 19.68 & 6 & 96 & 0.05 & 1 & 1.2 \\
\hline 94 & $27 / 02 / 1988$ & $05: 11: 14.75$ & 1.5 & 38.333 & 15.943 & 21.36 & 6 & 188 & 0.04 & 1.4 & 0.9 \\
\hline 95 & $17 / 04 / 1988$ & & 2.9 & & 15.982 & 60.65 & 9 & & 0.08 & 1.9 & 1.1 \\
\hline 96 & $02 / 05 / 1988$ & $02: 41: 02.81$ & 1.6 & 38.346 & 16.072 & 16.95 & 8 & 170 & 0.04 & 0.7 & 0.7 \\
\hline 97 & $15 / 06 / 1988$ & $00: 22: 02.52$ & 1.3 & 38.376 & 16.1 & 19.38 & 6 & 176 & 0.03 & 1.1 & 0.8 \\
\hline 98 & $17 / 07 / 1988$ & $02: 32: 13.27$ & 0.9 & 38.298 & 15.995 & 20.57 & 7 & 102 & 0.04 & 0.9 & 1.1 \\
\hline 99 & $17 / 07 / 1988$ & $17: 52: 28.66$ & 0.8 & 38.298 & 15.995 & 20.37 & 6 & 125 & 0.04 & 1.1 & 1.8 \\
\hline 100 & $19 / 07 / 1988$ & $09: 50: 24.37$ & 1.3 & 38.347 & 16.095 & 15.62 & 6 & 193 & 0.07 & 0.9 & 0.9 \\
\hline 101 & $21 / 07 / 1988$ & 10:18:59.62 & 1.3 & 38.396 & 16.058 & 16.23 & 7 & 166 & 0.05 & 0.9 & 0.7 \\
\hline 102 & $25 / 09 / 1988$ & 02:37:02.95 & 0.6 & 38.292 & 15.996 & 21.6 & 7 & 103 & 0.05 & 1 & 1.8 \\
\hline 103 & 18/11/1988 & 09:44:31.51 & 1.3 & 38.344 & 15.935 & 21.08 & 10 & 84 & 0.05 & 0.6 & 0.8 \\
\hline 104 & $22 / 12 / 1988$ & $11: 13: 50.37$ & 1.2 & 38.543 & 16.127 & 23.98 & 6 & 244 & 0.02 & 1.1 & 1.3 \\
\hline 105 & $11 / 01 / 1989$ & $11: 36: 36.58$ & 1.1 & 38.263 & 16.063 & 18.97 & 6 & 232 & 0.03 & 0.9 & 1.2 \\
\hline 106 & $29 / 01 / 1989$ & $19: 50: 47.89$ & 0.7 & 38.466 & 16.002 & 14.74 & 11 & 85 & 0.06 & 0.6 & 1.3 \\
\hline 107 & 07/03/1989 & $18: 52: 03.75$ & 1.0 & 38.293 & 16.055 & 18.66 & 6 & 161 & 0.05 & 1.1 & 1.3 \\
\hline
\end{tabular}


Table I. (continued).

\begin{tabular}{|c|c|c|c|c|c|c|c|c|c|c|c|}
\hline $\mathbf{N}^{\circ}$ & Date & Orig. Time & $\mathbf{M}_{\mathrm{L}}$ & Lat. $\mathbf{N}^{\circ}$ & Long. $\mathrm{E}^{\circ}$ & Depth & $\mathbf{N}$ & Gap & rms & Erh & Erz \\
\hline 108 & 09/03/1989 & $16: 41: 41.39$ & 1.5 & 38.442 & 16.179 & 19.29 & 7 & 235 & 0.06 & 0.9 & 0.8 \\
\hline 109 & $29 / 05 / 1989$ & 18:59:47.09 & 1.0 & 38.332 & 16.088 & 18.35 & 7 & 128 & 0.05 & 0.9 & 1.4 \\
\hline 110 & $30 / 05 / 1989$ & $00: 33: 46.14$ & 0.7 & 38.329 & 16.093 & 19.54 & 6 & 136 & 0.04 & . & 1.2 \\
\hline 111 & 04/06/1989 & $02: 20: 47.67$ & 1.1 & 38.332 & 16.092 & 19.15 & 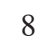 & 30 & 0.05 & 8 & 0.8 \\
\hline 112 & 04/06/1989 & $22: 36: 21.41$ & 1.5 & 38.332 & 16.091 & 19.11 & 9 & 130 & 0.06 & 0.7 & 0.7 \\
\hline 113 & 04/06/1989 & 22:58:02.94 & 1.0 & 38.331 & 16.093 & 19.45 & 9 & 132 & 0.06 & 0.7 & 0.7 \\
\hline 114 & 04/06/1989 & $23: 56: 18.07$ & 1.2 & 38.331 & 16.094 & 19.51 & 9 & 32 & 0.05 & 0.7 & 0.7 \\
\hline 115 & 04/06/1989 & $23: 57: 03.76$ & 1.6 & 38.333 & 16.094 & 19.65 & 10 & 130 & 0.06 & 0.7 & 0.7 \\
\hline 116 & 05/06/1989 & 04:09:21.01 & 1.6 & & 16.093 & 19.78 & 10 & 131 & 0.06 & 0.7 & 0.7 \\
\hline 117 & 06/06/1989 & 00:06:36.62 & 1.1 & & 16.097 & & 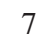 & & 0.08 & 0.9 & 0.9 \\
\hline 118 & $14 / 06 / 1989$ & 20:20:22.97 & 0.6 & & 16.095 & & 11 & & 0.07 & & 0.6 \\
\hline 119 & $14 / 06 / 1989$ & 21:01:00.28 & 0.8 & & 16.095 & & 9 & & 0.06 & & 0.7 \\
\hline 120 & $14 / 06 / 1989$ & $21: 18: 58.92$ & 1.5 & & & & 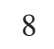 & & & & 0.8 \\
\hline 121 & $14 / 06 / 1989$ & & 0.7 & & & & . & & & & 0.7 \\
\hline 122 & $17 / 06 / 1989$ & 04:11:00.73 & 1.6 & 38.326 & 16.094 & 19.43 & $r$ & 144 & 0.04 & 0.8 & 0.8 \\
\hline 123 & $17 / 06 / 1989$ & $12: 37: 13.22$ & 0.9 & 38.334 & 16.095 & 19.82 & $r$ & 126 & 0.05 & 1.1 & 1 \\
\hline 124 & $24 / 06 / 1989$ & 01:03:57.11 & 0.5 & 38.326 & 16.09 & 19.49 & r & 140 & 0.04 & 0.8 & 0.8 \\
\hline 125 & $21 / 08 / 1989$ & $13: 52: 17.92$ & 1.3 & 38.421 & 16.018 & 15.42 & 6 & 155 & 0.02 & 1 & 1 \\
\hline 126 & $21 / 08 / 1989$ & $13: 58: 04.08$ & 1.1 & 38.423 & 16.018 & 15.78 & . & 157 & 0.03 & 0.9 & 0.7 \\
\hline 127 & $26 / 08 / 1989$ & 09:40:08.65 & 1.4 & 38.161 & 15.904 & 16.61 & 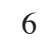 & 305 & 0.05 & 12 & 1.8 \\
\hline 128 & $13 / 12 / 1989$ & $18: 35: 49.30$ & 1.9 & 38.366 & 16.093 & 11.14 & 11 & 104 & 0.04 & 0.5 & 0.6 \\
\hline 129 & $21 / 12 / 1989$ & 09:00:32.11 & 1.5 & 38.270 & 16.082 & 18.8 & 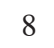 & 228 & 0.06 & 0.8 & 0.8 \\
\hline 130 & $21 / 12 / 1989$ & & 2.2 & 38.264 & & & 9 & 173 & 0.05 & 0.7 & 0.8 \\
\hline 131 & $21 / 12 / 1989$ & $12: 21: 35.23$ & 1.6 & & & & 7 & 230 & 0.06 & 0.9 & 1.2 \\
\hline 132 & $22 / 12 / 1989$ & 11:20:46.12 & 1.2 & & 16.009 & & 7 & 234 & 0.05 & 1 & 1.4 \\
\hline 133 & $31 / 01 / 1990$ & 21:38:04.83 & 1.2 & 38.494 & 16.03 & 21.58 & 7 & 116 & 0.02 & 1 & 1.3 \\
\hline 134 & $31 / 01 / 1990$ & $21: 38: 17.50$ & 1.8 & 38.491 & 16.024 & 21.71 & 10 & 111 & 0.03 & 0.6 & 0.9 \\
\hline 135 & $01 / 02 / 1990$ & $00: 40: 40.35$ & 2.1 & 38.489 & 16.025 & 21.85 & 11 & 109 & 0.04 & 0.6 & 0.8 \\
\hline 136 & 01/02/1990 & $00: 47: 27.58$ & 1.6 & 38.491 & 16.029 & & 11 & 113 & 0.03 & 0.7 & 0.7 \\
\hline 137 & 01/02/1990 & 01:31:18.08 & 1.5 & 38.489 & 16.026 & & 9 & 110 & 0.03 & 0.7 & 1 \\
\hline 138 & $01 / 02 / 1990$ & $03: 17: 57.13$ & 2.1 & 38.489 & & & 10 & 110 & 0.03 & 0.6 & 0.9 \\
\hline 139 & 01/02/1990 & $03: 25: 16.57$ & 1.3 & & & & 9 & 111 & 0.02 & 0.7 & 1 \\
\hline 140 & 01/02/1990 & 03:36:09.93 & 1.2 & 38.490 & 16.025 & 22.01 & 10 & 110 & 0.03 & 0.6 & 0.9 \\
\hline 141 & $01 / 02 / 1990$ & $04: 43: 30.58$ & 1.9 & 38.489 & 16.024 & 21.93 & 10 & 109 & 0.03 & 0.6 & 0.9 \\
\hline 142 & $01 / 02 / 1990$ & $04: 53: 20.05$ & 1.3 & 38.490 & 16.028 & 21.01 & 8 & 112 & 0.02 & 1.1 & 1.3 \\
\hline 143 & $01 / 02 / 1990$ & $05: 04: 25.49$ & 1.1 & 38.491 & 16.029 & 21.66 & 8 & 113 & 0.02 & 1.1 & 1.3 \\
\hline
\end{tabular}


Table I. (continued).

\begin{tabular}{|c|c|c|c|c|c|c|c|c|c|c|c|}
\hline $\mathbf{N}^{\circ}$ & Date & Orig. Time & $\mathbf{M}_{\mathbf{L}}$ & Lat. $\mathbf{N}^{\circ}$ & Long. $\mathrm{E}^{\circ}$ & Depth & $\mathbf{N}$ & Gap & rms & Erh & Erz \\
\hline 144 & 01/02/1990 & 05:46:02.16 & 2.1 & 38.489 & 16.023 & 22.23 & 11 & 109 & 0.04 & 0.6 & 0.9 \\
\hline 145 & 01/02/1990 & $06: 12: 50.19$ & 1.6 & 38.488 & 16.024 & 21.92 & 10 & 108 & .03 & 0.6 & 0.9 \\
\hline 146 & $01 / 02 / 1990$ & $06: 54: 18.82$ & 1.9 & 38.487 & 16.023 & 22.12 & 10 & 107 & 0.03 & 0.6 & 0.9 \\
\hline 147 & 01/02/1990 & $07: 46: 38.53$ & 1.6 & 38.489 & 16.024 & 22.53 & 8 & 109 & 0.03 & 0.7 & 1 \\
\hline 148 & 01/02/1990 & 08:56:54.99 & 1.6 & 38.475 & 16.018 & 16.04 & 10 & 95 & 0.07 & 0.5 & 1.2 \\
\hline 149 & 01/02/1990 & 18:44:58.38 & 2.0 & 38.489 & 16.024 & 21.89 & 12 & 109 & 0.04 & 0.6 & 0.8 \\
\hline 150 & $02 / 02 / 1990$ & $02: 48: 35.13$ & 2.0 & 38.490 & 16.024 & 21.35 & 12 & 110 & 0.05 & 0.6 & 0.8 \\
\hline 151 & $02 / 02 / 1990$ & 05:41:31.89 & 1.9 & 38.489 & 16.024 & 21.37 & 11 & 109 & 0.03 & 0.6 & 0.9 \\
\hline 152 & 02/02/1990 & $20: 12: 47.40$ & 1.1 & 38.494 & 16.033 & 21.18 & 8 & 118 & 0.02 & 0.9 & 1 \\
\hline 153 & 02/02/1990 & 23:01:36.98 & 0.6 & 38.391 & 15.999 & 13.24 & 10 & 62 & 0.05 & 0.4 & 0.6 \\
\hline 154 & 02/02/1990 & 23:01:57.77 & 0.8 & 38.394 & 16.006 & 13.49 & 6 & 154 & 0.03 & 0.8 & 0.7 \\
\hline 155 & 03/02/1990 & $23: 25: 23.83$ & 1.1 & 38.489 & 16.021 & 22.16 & 11 & 108 & 0.06 & 0.6 & 0.9 \\
\hline 156 & $11 / 02 / 1990$ & 21:02:30.34 & 1.1 & 38.498 & 16.026 & 22.65 & 6 & 193 & 0.02 & 1.1 & 1.8 \\
\hline 157 & $11 / 02 / 1990$ & 21:29:07.67 & 1.2 & & 16.031 & & 7 & 115 & 0.02 & 0.8 & 1.1 \\
\hline 158 & $28 / 02 / 1990$ & & 2.4 & 38.444 & & & 12 & 79 & & 0.6 & 0.7 \\
\hline 159 & $12 / 03 / 1990$ & 19:10:16.12 & 1.3 & 38.501 & & & 9 & & & 0.9 & 0.9 \\
\hline 160 & $16 / 05 / 1990$ & 21:33:08.97 & 1.1 & 38.245 & 15.976 & 21.14 & 6 & 216 & 0.05 & 1.1 & 1.3 \\
\hline 161 & $10 / 06 / 1990$ & 03:35:05.70 & 1.8 & 38.435 & 15.965 & 16.08 & 12 & 91 & 0.07 & 0.4 & 0.7 \\
\hline 162 & $10 / 07 / 1990$ & 20:03:40.77 & 1.0 & 38.297 & 16.085 & 20.56 & 7 & 188 & 0.06 & 0.9 & 1.1 \\
\hline 163 & $29 / 09 / 1990$ & $20: 39: 35.05$ & 2.2 & 38.382 & 16.165 & 16.06 & 11 & 218 & 0.08 & 0.7 & 0.6 \\
\hline 164 & 29/09/1990 & 21:39:11.39 & 2.5 & 38.388 & 16.136 & 11.97 & 11 & 181 & 0.07 & 0.5 & 1 \\
\hline 165 & $29 / 09 / 1990$ & $23: 47: 34.17$ & 1.1 & 38.392 & 16.177 & 12.82 & 6 & 228 & 0.17 & 1 & 1.1 \\
\hline 166 & $26 / 11 / 1990$ & 03:27:20.77 & 1.6 & 38.470 & 16.153 & 20 & 8 & 205 & 0.04 & 1.2 & 0.8 \\
\hline 167 & $10 / 12 / 1990$ & $04: 15: 15.48$ & 1.4 & 38.539 & 16.049 & 24.76 & 8 & 205 & 0.06 & 1.1 & 1 \\
\hline 168 & $31 / 01 / 1991$ & $06: 57: 55.05$ & 2.0 & 38.371 & 16.083 & 13.39 & 6 & 93 & 0.02 & 1 & 2.1 \\
\hline 169 & 10/03/1991 & $10: 35: 10.98$ & 1.7 & 38.432 & 16.148 & 9.12 & 10 & 197 & 0.12 & 1.1 & 1.2 \\
\hline 170 & 20/04/1991 & $03: 26: 20.14$ & 1.5 & 38.345 & 15.938 & 21.67 & 8 & 129 & 0.06 & 0.9 & 1.1 \\
\hline 171 & 24/09/1991 & $19: 52: 59.67$ & 1.6 & 38.564 & 16.041 & 18 & 6 & 201 & 0.06 & 1.5 & 2 \\
\hline 172 & 25/09/1991 & $20: 14: 56.94$ & 1.7 & 38.493 & 16.143 & 17.86 & 7 & 244 & 0.08 & 1.6 & 1 \\
\hline 173 & 29/11/1991 & $13: 54: 26.37$ & 1.6 & 38.257 & 15.958 & 10.35 & 6 & 186 & 0.07 & 1.9 & 2 \\
\hline 174 & 13/12/1991 & 21:51:57.84 & 1.5 & 38.564 & 15.983 & 15.82 & 7 & 149 & 0.07 & 1.4 & 1.4 \\
\hline 175 & $19 / 04 / 1992$ & $02: 35: 55.35$ & 2.2 & 38.283 & 16.061 & 19.86 & 12 & 187 & 0.07 & 0.7 & 0.7 \\
\hline 176 & $20 / 04 / 1992$ & $05: 19: 12.00$ & 2.0 & 38.277 & 16.059 & 19.97 & 10 & 197 & 0.07 & 0.7 & 0.8 \\
\hline 177 & $16 / 05 / 1992$ & $02: 22: 27.20$ & 1.9 & 38.334 & 16.039 & 57.34 & 9 & 99 & 0.04 & 1.7 & 1.1 \\
\hline 178 & $16 / 05 / 1992$ & $02: 52: 56.20$ & 1.4 & 38.483 & 16.049 & 21.73 & 10 & 112 & 0.02 & 0.6 & 0.8 \\
\hline 179 & $23 / 05 / 1992$ & 01:04:56.21 & 2.3 & 38.470 & 15.948 & 13.59 & 12 & 105 & 0.12 & 0.6 & 0.9 \\
\hline
\end{tabular}


Table I. (continued).

\begin{tabular}{|c|c|c|c|c|c|c|c|c|c|c|c|}
\hline $\mathbf{N}^{\circ}$ & Date & Orig. Time & $\mathbf{M}_{\mathbf{L}}$ & Lat. $\mathrm{N}^{\circ}$ & Long. $\mathrm{E}^{\circ}$ & Depth & $\mathbf{N}$ & Gap & rms & Erh & Erz \\
\hline 180 & 08/07/1992 & $05: 35: 54.28$ & 2.1 & 38.287 & 16.045 & 16.95 & 11 & 187 & 0.14 & 1.5 & 0.8 \\
\hline 181 & 02/08/1992 & 00:03:15.14 & 1.2 & 74 & 99 & 14 & 6 & 139 & 0.14 & .2 & 2.1 \\
\hline 182 & 03/08/1992 & $04: 37: 14.52$ & 1.9 & 38.280 & 16.013 & 13.24 & 11 & 103 & 0.14 & 0.7 & 0.9 \\
\hline 183 & $10 / 08 / 1992$ & $22: 12: 19.42$ & 1.6 & 38.290 & 15.997 & 15.45 & 11 & 105 & 0.08 & 0.6 & 1.1 \\
\hline 184 & $12 / 08 / 1992$ & $03: 36: 52.20$ & 0.7 & 38.343 & 16.081 & 9.87 & 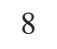 & 106 & 0.04 & 0.9 & 2.1 \\
\hline 185 & $11 / 09 / 1992$ & $12: 05: 31.43$ & 2.2 & 38.280 & 16.029 & 12.28 & 11 & 135 & 0.09 & .8 & 1.2 \\
\hline 186 & $11 / 09 / 1992$ & $21: 16: 48.29$ & 1.3 & 38.273 & 16.033 & 59 & 8 & 160 & 0.07 & 0.8 & 1.4 \\
\hline 187 & $11 / 09 / 1992$ & $21: 30: 43.36$ & 2.1 & 38.262 & 16.04 & 15.39 & 12 & 220 & 0.07 & 0.6 & 0.8 \\
\hline 188 & $11 / 09 / 1992$ & $21: 34: 12.69$ & 1.5 & 38.259 & 16.038 & 12.8 & 12 & 203 & 0.13 & 0.6 & 0.8 \\
\hline 189 & $11 / 09 / 1992$ & $21: 36: 46.91$ & 2.0 & 38.265 & 16.043 & 13.54 & 11 & 218 & 0.08 & .8 & 0.8 \\
\hline 190 & $11 / 09 / 1992$ & $21: 40: 37.08$ & 1.7 & 66 & 34 & 12.5 & 10 & 205 & 0.1 & .8 & 1.3 \\
\hline 191 & $11 / 09 / 1992$ & $21: 44: 12.83$ & 1.6 & & 16.039 & 1 & 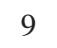 & 208 & 0.06 & 9 & 1.3 \\
\hline 192 & $11 / 09 / 1992$ & 21:54:18.75 & 1.0 & & & & . & 198 & & 2 & 1.7 \\
\hline 193 & 11/09/1992 & 21:59:09.57 & 1.1 & 38.270 & 16.042 & 16.61 & 10 & 195 & 0.14 & 0.6 & 1 \\
\hline 194 & $11 / 09 / 1992$ & $22:$ & 2.1 & 76 & 16 & 12 & 11 & 139 & 0.09 & 0.8 & 1.2 \\
\hline 195 & $11 / 09 / 1992$ & $22: 01: 23.17$ & 1.6 & 38 & & 14 & 1 & 221 & 0.07 & 0.9 & .3 \\
\hline 196 & $11 / 09 / 1992$ & $22: 25: 24.05$ & 1.5 & 38.262 & 16.038 & 14 & . & 219 & 0.06 & 0.9 & 1.3 \\
\hline 197 & 11/09/1992 & $22: 53: 51.68$ & 1.1 & 38.268 & 16.04 & 13.65 & 9 & 204 & 0.07 & 0.8 & 0.9 \\
\hline 198 & 11/09/1992 & 23:00:46.96 & 0.7 & 38.319 & 16.016 & 1.86 & r & 97 & 0.21 & 0.9 & 1.4 \\
\hline 199 & $11 / 09 / 1992$ & 23:58:05.50 & 1.0 & 38.280 & 16.028 & 11.18 & 7 & 132 & 0.07 & 1.4 & 2.3 \\
\hline 200 & $12 / 09 / 1992$ & 00:02:07.72 & 1.0 & 38.262 & 16.041 & 14.91 & 9 & 221 & 0.07 & 0.9 & 1.3 \\
\hline 201 & $12 / 09 / 1992$ & 00:09:17.40 & 0.8 & 38.263 & 16.04 & 13.29 & 9 & 219 & 0.09 & 0.4 & 0.8 \\
\hline 202 & $12 / 09 / 1992$ & $00: 48: 10.56$ & 0.5 & 38.319 & 16.015 & 1.83 & 6 & 134 & 0.22 & 0.9 & 1.6 \\
\hline 203 & $12 / 09 / 1992$ & 01:58:10.93 & 1.0 & 38.280 & 16.028 & 10.81 & 8 & 131 & 0.07 & 1.2 & 1.6 \\
\hline 204 & $12 / 09 / 1992$ & $03: 03: 25.90$ & 0.9 & & 16.036 & & 7 & 196 & 0.06 & 1 & 1.7 \\
\hline 205 & $12 / 09 / 1992$ & $03: 29: 06.56$ & 1.1 & 38.267 & 16.044 & 12.48 & 10 & 211 & 0.09 & 0.8 & 0.7 \\
\hline 206 & $12 / 09 / 1992$ & $04: 27: 17.23$ & 1.3 & 38.270 & & & 9 & 198 & 0.09 & 0.8 & 0.7 \\
\hline 207 & $12 / 09 / 1992$ & $05: 37: 42.28$ & 1.2 & 38.261 & 16.037 & 14.29 & 9 & 220 & 0.07 & 0.9 & 1.3 \\
\hline 208 & $12 / 09 / 1992$ & $05: 38: 21.74$ & 1.2 & 38.320 & 16.02 & 0.01 & 7 & 97 & 0.25 & 0.7 & 2.5 \\
\hline 209 & $12 / 09 / 1992$ & 05:38:36.19 & 1.4 & 38.258 & 16.038 & 14.48 & 9 & 223 & 0.07 & 0.9 & 1.3 \\
\hline 210 & $12 / 09 / 1992$ & $06: 15: 30.92$ & 0.9 & 38.316 & 16.025 & 0.85 & 6 & 101 & 0.21 & 0.4 & 3 \\
\hline 211 & $12 / 09 / 1992$ & $06: 35: 34.98$ & 1.1 & 38.319 & 16.019 & 0.05 & 7 & 97 & 0.24 & 0.7 & 2.3 \\
\hline 212 & $12 / 09 / 1992$ & 07:22:15.96 & 0.8 & 38.320 & 16.019 & 0.01 & 7 & 97 & 0.25 & 0.8 & 2.7 \\
\hline 213 & $12 / 09 / 1992$ & 10:02:11.58 & 0.8 & 38.270 & 16.04 & 14.14 & 6 & 195 & 0.06 & 1.1 & 1.6 \\
\hline 214 & $18 / 09 / 1992$ & $01: 23: 12.64$ & 1.5 & 38.272 & 16.031 & 12.55 & 10 & 155 & 0.07 & 0.8 & 1.3 \\
\hline 215 & $18 / 09 / 1992$ & $03: 56: 35.45$ & 1.0 & 38.269 & 16.034 & 13.22 & 8 & 183 & 0.05 & 0.9 & 1.4 \\
\hline
\end{tabular}


Table I. (continued).

\begin{tabular}{|c|c|c|c|c|c|c|c|c|c|c|c|}
\hline $\mathbf{N}^{\circ}$ & Date & Orig. Time & $\mathbf{M}_{\mathbf{L}}$ & Lat. $\mathbf{N}^{\circ}$ & Long. $\mathrm{E}^{\circ}$ & Depth & $\mathbf{N}$ & Gap & rms & Erh & Erz \\
\hline 216 & 18/09/1992 & 04:48:24.98 & 1.5 & 38.273 & 16.032 & 12.56 & 9 & 157 & 0.06 & 0.8 & 1.3 \\
\hline 217 & $19 / 09 / 1992$ & $14: 31: 17.14$ & 1.2 & 38.317 & 16.051 & 15.38 & 8 & 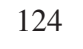 & 0.04 & 0.8 & 1.4 \\
\hline 218 & 29/10/1992 & $21: 16: 32.60$ & 1.3 & 38.382 & 16.14 & 14.76 & 10 & . & 0.05 & 0.7 & 1.3 \\
\hline 219 & 11/11/1992 & 04:48:34.60 & 1.0 & 38.502 & 15.958 & 13.22 & 6 & 133 & 0.03 & 1 & 2 \\
\hline 220 & $04 / 12 / 1992$ & $19: 32: 02.12$ & 0.5 & 38.440 & 16.014 & 11.55 & 6 & 17 & 0.06 & 1.3 & 3 \\
\hline 221 & 07/12/1992 & 04:06:00.46 & 1.5 & 38.272 & 16.031 & 14.58 & 10 & 156 & 0.1 & 0.7 & 0.8 \\
\hline 222 & $16 / 01 / 1993$ & $13: 20: 39.96$ & 0.5 & 38.275 & 16.013 & 12.82 & 6 & 118 & 0.05 & 1.2 & 2.1 \\
\hline 223 & 06/03/1993 & 09:07:16.22 & 2.2 & 38.416 & 16.201 & 15.27 & 8 & 248 & 0.06 & 0.9 & 1.5 \\
\hline 224 & $10 / 04 / 1993$ & 20:50:10.97 & 1.9 & 38.337 & 16.12 & 17.11 & 11 & 117 & 0.07 & 0.7 & 1.2 \\
\hline 225 & $23 / 04 / 1993$ & 02:46:32.05 & 1.0 & 38.438 & 15.919 & 12.98 & 10 & 152 & 0.1 & 0.8 & 1.4 \\
\hline 226 & $11 / 05 / 1993$ & 06:36:07.15 & 1.2 & 38.302 & 15.932 & 16.31 & 10 & 88 & 0.09 & 0.8 & 1.1 \\
\hline 227 & 02/06/1993 & $08: 26: 14.41$ & 2.1 & 38.535 & 16.053 & 17.35 & 10 & 174 & 0.06 & 0.6 & 1.4 \\
\hline 228 & $24 / 06 / 1993$ & 05:50:10.83 & 1.3 & 38.313 & 16.023 & 16.13 & 6 & 138 & .03 & 0.9 & 1.6 \\
\hline 229 & $30 / 06 / 1993$ & $21: 20: 27.01$ & 2.0 & 38.342 & 15.94 & 16.46 & 10 & 105 & 0.09 & 0.6 & 1.2 \\
\hline 230 & $30 / 06 / 1993$ & 22:07:50.91 & 1.8 & 38.339 & 15.938 & 16.94 & 9 & 1 & 0.09 & 0.7 & 1.2 \\
\hline 231 & $30 / 06 / 1993$ & $23: 49: 35.53$ & 0.7 & 38.345 & 15.934 & 16.88 & 6 & & 0.05 & 1.1 & 1.6 \\
\hline 232 & 01/07/1993 & 18:52:19.12 & 1.1 & 38.343 & 37 & 1 & 7 & 14 & & 1.1 & 1.6 \\
\hline 233 & 01/07/1993 & 19:01:49.32 & 2.1 & 38.343 & 15.938 & 16.49 & 11 & 81 & 0.08 & 0.6 & 1.2 \\
\hline 234 & 01/07/1993 & 19:43:11.91 & 1.1 & 38.342 & 15.937 & 16.96 & 9 & 104 & 0.06 & 0.9 & 1.4 \\
\hline 235 & 01/07/1993 & $20: 29: 50.40$ & 1.1 & 38.341 & 15.936 & 17.02 & 7 & 149 & 0.06 & 1.1 & 1.6 \\
\hline 236 & 01/07/1993 & $20: 34: 56.45$ & 1.3 & 38.341 & 15.941 & 17.82 & 8 & 150 & 0.04 & 0.6 & 1.5 \\
\hline 237 & 01/07/1993 & 21:06:47.01 & 1.4 & 337 & 15.939 & 17 & 10 & 15 & 0.09 & 1 & 1.5 \\
\hline 238 & 01/07/1993 & $21: 41: 53.12$ & 1.4 & 38.343 & 15.94 & 16.41 & 11 & 79 & 0.08 & 0.6 & 1.2 \\
\hline 239 & 01/07/1993 & $22: 55: 17.24$ & 1.5 & 38.337 & 15.94 & 17.41 & 10 & 155 & 0.08 & 1 & 1.5 \\
\hline 240 & 02/07/1993 & $00: 18: 23.24$ & 1.3 & 38.347 & 15.946 & 15.78 & 11 & 105 & 0.1 & 0.6 & 1.2 \\
\hline 241 & 02/07/1993 & $00: 20: 12.80$ & 1.1 & 38.335 & 15.933 & 17.52 & 8 & 154 & 0.08 & 1 & 1.5 \\
\hline 242 & 02/07/1993 & 01:03:54.61 & 1.9 & 38.344 & 15.941 & 16.53 & 12 & 79 & 0.09 & 0.6 & 1.2 \\
\hline 243 & 02/07/1993 & 04:16:11.71 & 1.3 & 38.346 & 15.942 & 16.42 & 9 & 132 & 0.07 & 0.7 & 1.2 \\
\hline 244 & 02/07/1993 & $12: 40: 16.91$ & 0.9 & 38.364 & 15.974 & 0.45 & 6 & 134 & 0.19 & 0.4 & 3 \\
\hline 245 & 02/07/1993 & $13: 55: 44.29$ & 1.1 & 38.363 & 15.975 & 0.09 & 6 & 136 & 0.17 & 0.5 & 1.5 \\
\hline 246 & 02/07/1993 & $16: 17: 51.98$ & 1.5 & 38.326 & 16.018 & 11.92 & 10 & 89 & 0.05 & 0.5 & 1.3 \\
\hline 247 & $15 / 07 / 1993$ & 10:18:04.47 & 2.0 & 38.452 & 16.11 & 14.21 & 10 & 138 & 0.07 & 0.6 & 1.2 \\
\hline 248 & $12 / 08 / 1993$ & 01:03:41.90 & 0.5 & 38.275 & 16.035 & 12.79 & 6 & 161 & 0.04 & 1.3 & 1.9 \\
\hline 249 & $13 / 08 / 1993$ & $20: 54: 21.18$ & 0.6 & 38.276 & 16.035 & 12.51 & 8 & 161 & 0.08 & 0.8 & 1.4 \\
\hline 250 & $13 / 09 / 1993$ & $11: 51: 20.14$ & 0.9 & 38.299 & 16.04 & 16.04 & 8 & 133 & 0.19 & 0.8 & 1.4 \\
\hline 251 & $18 / 12 / 1993$ & 06:53:57.30 & 1.9 & 38.432 & 15.941 & 16.42 & 10 & 115 & 0.12 & 0.5 & 0.8 \\
\hline 252 & $13 / 03 / 1994$ & $14: 02: 49.43$ & 2.8 & 38.450 & 15.891 & 43.59 & 10 & 199 & 0.1 & 1.5 & 1.4 \\
\hline
\end{tabular}


(1935) procedure (Di Grazia et al., 2001). The estimated $M_{L}$ ranges between 0.5 and 3.0.

\section{Data analysis and results}

\subsection{Focal mechanisms and stress tensor analysis}

Fault plane solutions (FPS) were determined using the FPFIT program (Reasenberg and Oppenheimer, 1985). The FPFIT program constrains the mechanism to be double-couple and performs a grid search over the available solution space. In order to obtain good azimuthal data coverage for each focal mechanism solution, seismograms were reread to check polarities and a minimum of 7 impulsive Pwave first motions in different azimuth (9 on average) were considered for each selected event. The final dataset consists of 120 wellconstrained fault plane solutions (figs. 2 and 3; table II). In particular, 53\% normal faulting, $46 \%$ strike slip faulting, and $2 \%$ reverse faulting mechanisms have been identified. The FPFIT program output also gives $\mathrm{P}$ - and T- axes orientation. The spatial distribution of deformation axes (fig. 2) shows for the P-axes a strong dispersion with a maximum in the $\mathrm{N} 40^{\circ}-60^{\circ} \mathrm{E}$ class. Conversely, the prevalence of the T-axes in the $\mathrm{N} 300^{\circ}-320^{\circ} \mathrm{E}$ is well evidenced.

To determine stress directions from fault plane solutions, we used the Focal Mechanism Stress Inversion (FMSI) computer program developed by Gephart and Forsyth (1984) and Gephart (1990). This method is based on the following basic assumptions: 1) stress is uniform in the rock volume of the seismic sample investigated; 2) earthquakes are shear dislocations on pre-existing faults; 3 ) slip occurs in the direction of the resolved shear stress on the fault plane. Four stress parameters are calculated: three of them define the orientations of the main stress axes $\sigma_{1}, \sigma_{2}$, and $\sigma_{3}$, the other is a measure of relative stress magnitude $R=\left(\sigma_{2}-\sigma_{l}\right) /\left(\sigma_{3^{-}}-\sigma_{l}\right)$. Moreover, a variable misfit $(F)$ is introduced to define discrepancies between the stress tensor and the observed fault plane solutions. For a given stress model, the misfit of a single focal mechanism is defined as the smallest rotation around any arbitrary axis which brings one of the nodal planes, its slip direction and the sense of slip into an orientation that is consistent with the stress model. Each FPS receives two misfits, one for each nodal plane. If an a priori choice of the fault plane is not made, the nodal plane with the smallest misfit is assumed as the fault plane. The size of the average misfit provides a guide of how well the assumption of stress homogeneity is fulfilled in relation to the seismic sample submitted to the inversion algorithm (Michael, 1987). In the light of the results from a series of tests carried out by Wyss et al. (1992), Gillard et al. (1996), Cocina et al. (1997) to identify the relationship between FPS uncertainties and average misfit in the case of uniform stress, we assume that the condition of a homogeneous stress distribution is fulfilled if the misfit, $F$, is smaller than $6^{\circ}$ and that it is not fulfilled if $F>9^{\circ}$. For $F$ values between $6^{\circ}$ and $9^{\circ}$ the solution is considered acceptable, but it may reflect some heterogeneity. The statistical confidence limits established for possible stress orientations that are consistent with the observed focal mechanisms may give an additional contribution because they generally tend to enlarge for increasing stress heterogeneity (Cocina et al., 1997). We computed the 90\% confidence level using the statistical procedure described by Parker and McNutt (1980) and Gephart and Forsyth (1984). The size of the $90 \%$ confidence limits will not be a criterion for preferring an inversion result, because it does not measure the quality of the result but rather the degree to which it is constrained.

In the present study, the Gephart and Forsyth (1984) inversion algorithm was applied to the 120 well-constrained fault plane solutions. If they are produced by a single stress tensor, then the variety among the fault plane solutions may be the result of the presence of planes of weakness with different orientations to accommodate the slip. On the other hand, the variation could reflect the inhomogeneity of stress within the crust.

Each of the FPS was assigned a weight ( 1 =sufficiently constrained, $2=$ well constrained) based on a qualitative evaluation of the polarity distribution and score. Fault parameter uncer- 


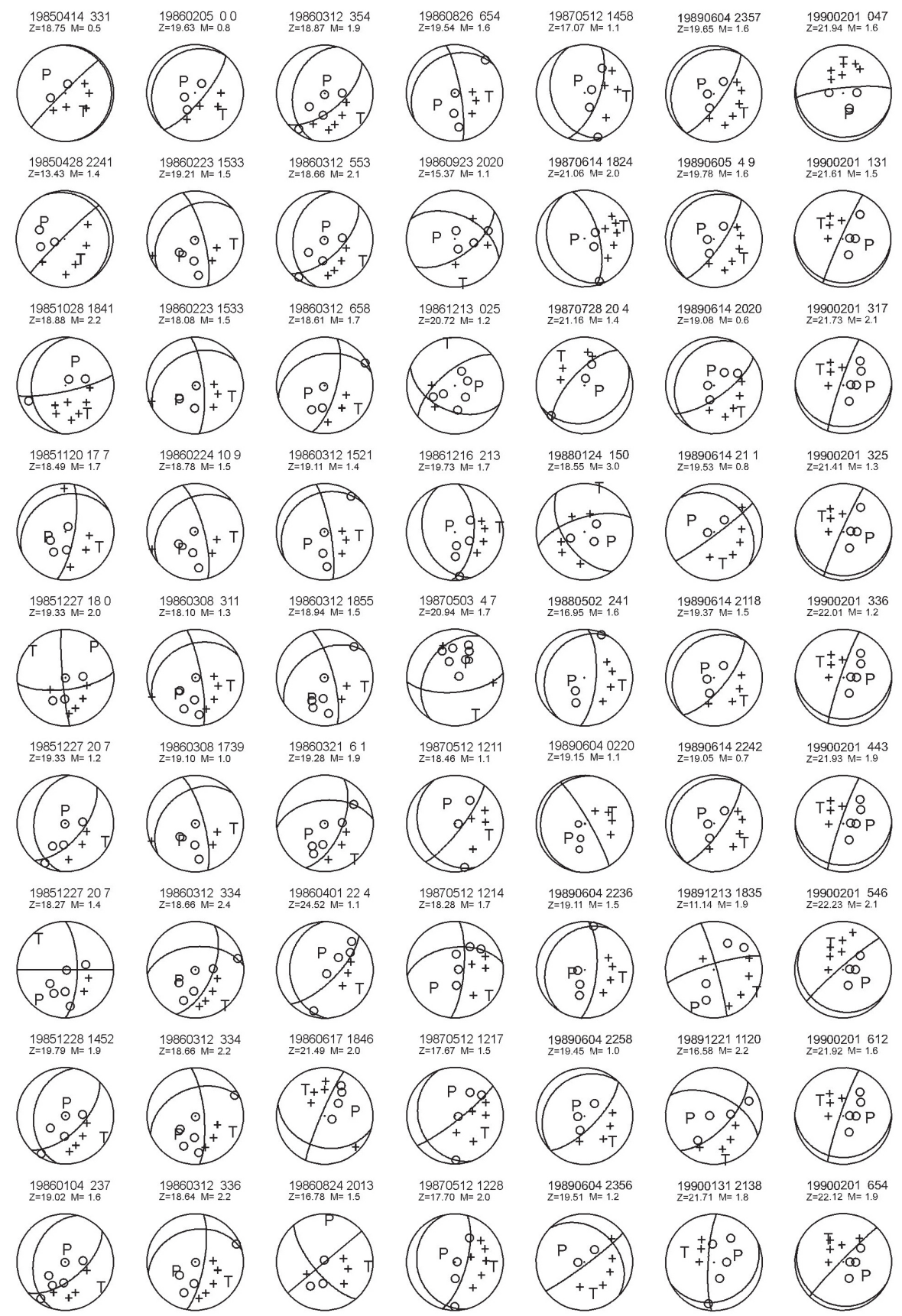

Fig. 2. Fault plane solutions (lower hemisphere equal-area projection) of the 120 best constrained events. For each mechanism the date (year, month, day), the origin time (hour, minute), the focal depth in $\mathrm{km}(\mathrm{z})$ and the magnitude $\left(\mathrm{M}_{\mathrm{L}}\right)$ are reported. $\mathrm{P}$ and $\mathrm{T}$ denote the $\mathrm{P}$ - and T-axes position. Open circles and crosses indicate dilatations and compressions, respectively. 
Fig. 2. (continued)

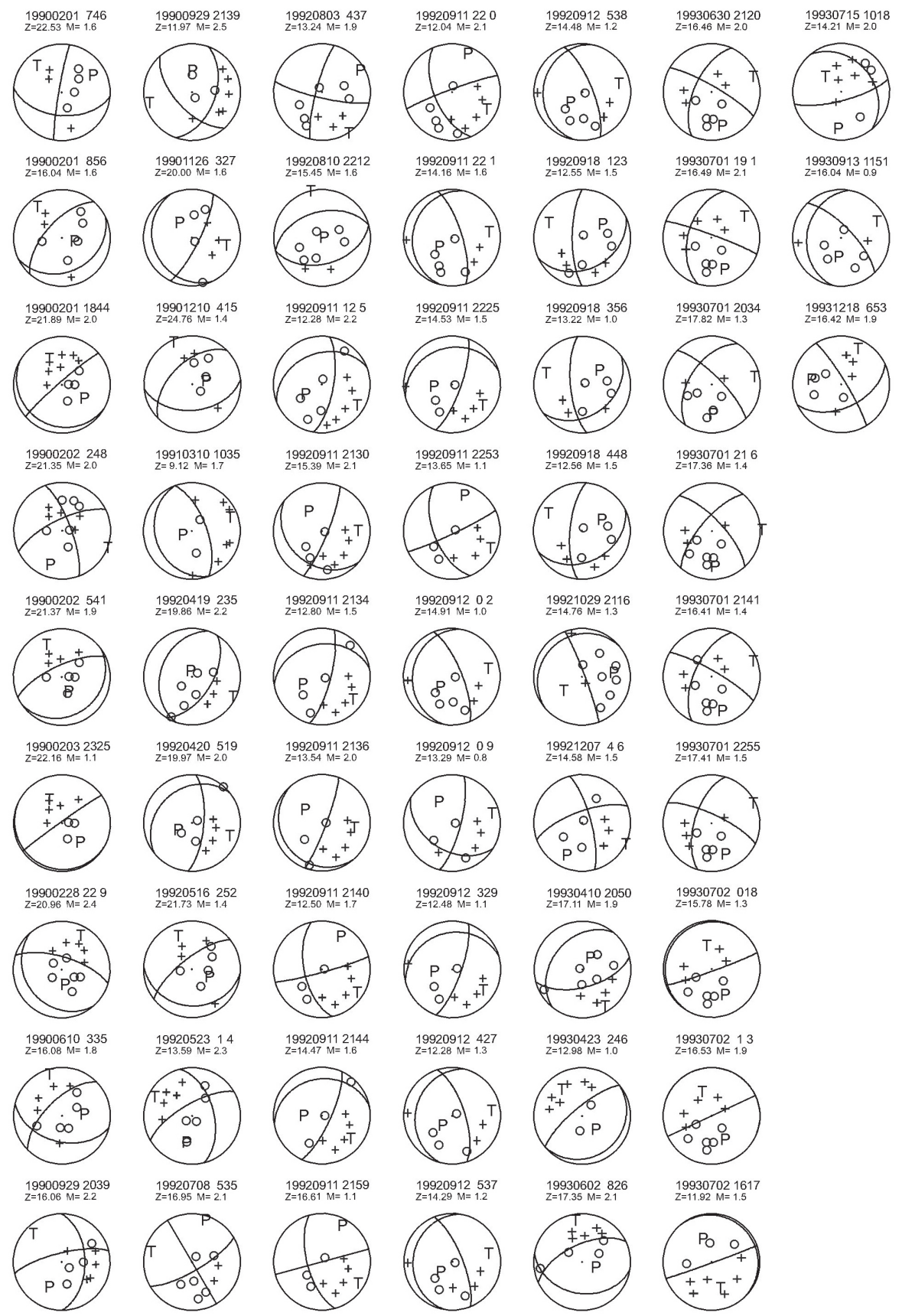


Table II. Focal parameters of the 120 selected events. Strike, Dip, Rake, Azimuth and Plunge are given in degrees. $\Delta$ is the error referred to the nodal plane.

Paxis

Taxis

\begin{tabular}{|c|c|c|c|c|c|c|c|c|c|c|c|c|c|}
\hline $\mathbf{N}^{\circ}$ & Date & Orig. Time & $\mathbf{N}_{\mathrm{p}}$ & Strike & Dip & Rake & $\Delta \mathrm{Str}$ & $\Delta$ Dip & $\Delta$ Rake & Azm & Plunge & Azm & Plunge \\
\hline 1 & $14 / 04 / 1985$ & 03:31:22.04 & 7 & 355 & 5 & 40 & 15 & 10 & 15 & 311 & 42 & 139 & 48 \\
\hline 4 & 28/04/1985 & $22: 41: 03.14$ & 8 & 5 & 10 & 20 & 13 & 13 & 10 & 306 & 41 & 145 & 48 \\
\hline 12 & $28 / 10 / 1985$ & 18:41:47.79 & 10 & 185 & 30 & -160 & 13 & 10 & 10 & 17 & 47 & 145 & 29 \\
\hline 14 & 20/11/1985 & $17: 07: 22.24$ & 8 & 10 & 75 & -110 & 10 & 10 & 20 & 255 & 56 & 116 & 27 \\
\hline 18 & $27 / 12 / 1985$ & 18:00:10.63 & 9 & 175 & 85 & -160 & 3 & 3 & 10 & 41 & 18 & 307 & 10 \\
\hline 20 & 27/12/1985 & $20: 07: 31.85$ & 8 & 185 & 35 & -120 & 20 & 10 & 10 & 350 & 69 & 116 & 13 \\
\hline 21 & $27 / 12 / 1985$ & 20:07:49.31 & 8 & 270 & 90 & -20 & 5 & 10 & 10 & 223 & 14 & 317 & 14 \\
\hline 22 & $28 / 12 / 1985$ & $14: 52: 24.25$ & 9 & 190 & 35 & -120 & 20 & 10 & 10 & 355 & 69 & 121 & 13 \\
\hline 24 & 04/01/1986 & $02: 37: 06.54$ & 10 & 195 & 35 & -120 & 20 & 8 & 10 & 360 & 69 & 126 & 13 \\
\hline 27 & 05/02/1986 & 00:00:30.34 & 7 & 40 & 75 & -90 & 13 & 5 & 20 & 310 & 60 & 130 & 30 \\
\hline 28 & $23 / 02 / 1986$ & $15: 33: 29.42$ & 7 & 345 & 75 & -120 & 10 & 10 & 20 & 221 & 51 & 98 & 24 \\
\hline 29 & 23/02/1986 & $15: 33: 47.76$ & 7 & 355 & 75 & -120 & 20 & 13 & 20 & 231 & 51 & 108 & 24 \\
\hline 31 & $24 / 02 / 1986$ & 10:09:56.51 & 7 & 345 & 75 & -120 & 10 & 10 & 20 & 221 & 51 & 98 & 24 \\
\hline 32 & 08/03/1986 & 03:11:28.64 & 10 & 350 & 80 & -120 & 5 & 8 & 20 & 229 & 47 & 104 & 29 \\
\hline 33 & 08/03/1986 & 17:39:09.65 & 7 & 345 & 75 & -120 & 13 & 10 & 20 & 221 & 51 & 98 & 24 \\
\hline 34 & $12 / 03 / 1986$ & 03:34:09.64 & 10 & 265 & 50 & -40 & 10 & 20 & 10 & 240 & 53 & 142 & 6 \\
\hline 35 & $12 / 03 / 1986$ & 03:34:13.93 & 9 & 355 & 75 & -130 & 3 & 8 & 20 & 225 & 45 & 114 & 20 \\
\hline 36 & $12 / 03 / 1986$ & 03:36:04.43 & 10 & 10 & 75 & -120 & 8 & 10 & 20 & 246 & 51 & 123 & 24 \\
\hline 38 & $12 / 03 / 1986$ & 03:54:00.19 & 10 & 195 & 35 & -120 & 18 & 8 & & & 69 & 126 & 13 \\
\hline 41 & $12 / 03 / 1986$ & 05:53:25.81 & 9 & 190 & 35 & -120 & 18 & 10 & 10 & 355 & 69 & 121 & 13 \\
\hline 42 & $12 / 03 / 1986$ & $06: 58: 59.54$ & 7 & 15 & 70 & -120 & 13 & 13 & 20 & 247 & 55 & 127 & 19 \\
\hline 44 & $12 / 03 / 1986$ & $15: 21: 38.95$ & 7 & 350 & 80 & -110 & 20 & 13 & 20 & 237 & 51 & 97 & 32 \\
\hline 45 & $12 / 03 / 1986$ & 18:55:09.54 & 8 & 345 & 75 & -130 & 13 & 10 & 20 & 215 & 45 & 104 & 20 \\
\hline 46 & $21 / 03 / 1986$ & 06:01:18.65 & 9 & 265 & 50 & -40 & 13 & 20 & 10 & 240 & 53 & 142 & 6 \\
\hline 47 & 01/04/1986 & 22:04:28.45 & 8 & 45 & 70 & -70 & 5 & 5 & 20 & 344 & 60 & 120 & 22 \\
\hline 49 & 17/06/1986 & $18: 46: 00.44$ & 9 & 105 & 35 & -10 & 10 & 20 & 20 & 81 & 41 & 321 & 31 \\
\hline 57 & 24/08/1986 & $20: 13: 25.54$ & 7 & 140 & 70 & -180 & 10 & 13 & 5 & 3 & 14 & 97 & 14 \\
\hline 58 & 26/08/1986 & $06: 54: 18.47$ & 7 & 350 & 80 & -110 & 20 & 10 & 20 & 237 & 51 & 97 & 32 \\
\hline 60 & 23/09/1986 & $20: 20: 35.27$ & 7 & 55 & 60 & -130 & 5 & 5 & 10 & 272 & 55 & 172 & 7 \\
\hline 65 & $13 / 12 / 1986$ & $00: 25: 35.03$ & 8 & 230 & 65 & -130 & 10 & 13 & 5 & 92 & 52 & 348 & 11 \\
\hline 67 & $16 / 12 / 1986$ & 02:13:16.01 & 9 & 160 & 35 & -110 & 10 & 10 & 20 & 310 & 74 & 84 & 11 \\
\hline 72 & 03/05/1987 & 04:07:40.12 & 8 & 85 & 65 & -60 & 20 & 5 & 5 & 38 & 59 & 154 & 15 \\
\hline 75 & $12 / 05 / 1987$ & $12: 11: 15.13$ & 7 & 155 & 25 & -150 & 13 & 20 & 15 & 333 & 52 & 110 & 29 \\
\hline 77 & $12 / 05 / 1987$ & $12: 14: 59.85$ & 9 & 5 & 75 & -140 & 18 & 8 & 10 & 231 & 38 & 129 & 15 \\
\hline
\end{tabular}


Table II. (continued).

Paxis

Taxis

\begin{tabular}{|c|c|c|c|c|c|c|c|c|c|c|c|c|c|}
\hline $\mathbf{N}^{\circ}$ & Date & Orig. Time & $\mathbf{N}_{\mathrm{p}}$ & Strike & Dip & Rake & $\Delta \mathrm{Str}$ & $\Delta$ Dip & $\Delta$ Rake & Azm & Plunge & Azm & Plunge \\
\hline 78 & $12 / 05 / 1987$ & $12: 17: 39.33$ & 9 & 160 & 25 & -160 & 3 & 10 & 5 & 347 & 48 & 122 & 33 \\
\hline 80 & $12 / 05 / 1987$ & $12: 28: 38.11$ & 10 & 15 & 70 & -80 & 8 & 0 & 20 & 301 & 64 & 97 & 24 \\
\hline 81 & $12 / 05 / 1987$ & 14:58:19.51 & 8 & 20 & 65 & -70 & 8 & 3 & 20 & 324 & 64 & 95 & 18 \\
\hline 84 & $14 / 06 / 1987$ & $18: 24: 08.19$ & 9 & 155 & 25 & -100 & 20 & 5 & 20 & 265 & 69 & 73 & 20 \\
\hline 85 & $28 / 07 / 1987$ & 20:04:14.44 & 7 & 65 & 25 & -70 & 10 & 5 & 10 & 116 & 67 & 320 & 21 \\
\hline 90 & $24 / 01 / 1988$ & 01:50:50.12 & 8 & 145 & 55 & -30 & 20 & 5 & 10 & 113 & 44 & 17 & 7 \\
\hline 96 & 02/05/1988 & 02:41:02.81 & 7 & 5 & 70 & -100 & 20 & 13 & 20 & 259 & 64 & 103 & 24 \\
\hline 111 & 04/06/1989 & 02:20:47.67 & 7 & 190 & 10 & -50 & 20 & 20 & 20 & 234 & 48 & 70 & 38 \\
\hline 112 & 04/06/1989 & $22: 36: 21.41$ & 8 & 5 & 70 & -100 & 18 & 10 & 20 & 259 & 64 & 103 & 24 \\
\hline 113 & 04/06/1989 & 22:58:02.94 & 7 & 45 & 70 & -90 & 13 & 5 & 20 & 315 & 65 & 135 & 25 \\
\hline 114 & 04/06/1989 & $23: 56: 18.07$ & 7 & 315 & 30 & -10 & 20 & 10 & 10 & 295 & 42 & 168 & 33 \\
\hline 115 & 04/06/1989 & 23:57:03.76 & 9 & 35 & 75 & -90 & 8 & 5 & 20 & 305 & 60 & 125 & 30 \\
\hline 116 & 05/06/1989 & 04:09:21.01 & 9 & 35 & 75 & -90 & 8 & 5 & 20 & 305 & 60 & 125 & 30 \\
\hline 118 & $14 / 06 / 1989$ & $20: 20: 22.97$ & 9 & 55 & 70 & -80 & 8 & 3 & 20 & 341 & 64 & 137 & 24 \\
\hline 119 & $14 / 06 / 1989$ & 21:01:00.28 & 7 & 315 & 30 & -10 & 20 & 10 & 10 & 295 & 42 & 168 & 33 \\
\hline 120 & $14 / 06 / 1989$ & 21:18:58.92 & 7 & 40 & 70 & -90 & 13 & 5 & 20 & 310 & 65 & 130 & 25 \\
\hline 121 & $14 / 06 / 1989$ & $22: 42: 35.30$ & 8 & 35 & 75 & -90 & 8 & 5 & 20 & 305 & 60 & 125 & 30 \\
\hline 128 & $13 / 12 / 1989$ & $18: 35: 49.30$ & 9 & 345 & 85 & -170 & 3 & 13 & 20 & 210 & 11 & 119 & 3 \\
\hline 130 & $21 / 12 / 1989$ & $11: 20: 23.95$ & 8 & 45 & 65 & -140 & 8 & 5 & 10 & 265 & 45 & 168 & 6 \\
\hline 134 & $31 / 01 / 1990$ & $21: 38: 17.50$ & 8 & 65 & 20 & -30 & 18 & 18 & 20 & 73 & 52 & 288 & 33 \\
\hline 136 & $01 / 02 / 1990$ & $00: 47: 27.58$ & 9 & 105 & 10 & -70 & 20 & 10 & 20 & 171 & 54 & 358 & 36 \\
\hline 137 & $01 / 02 / 1990$ & 01:31:18.08 & 8 & 95 & 10 & -20 & 20 & 20 & 20 & 105 & 48 & 304 & 41 \\
\hline 138 & $01 / 02 / 1990$ & $03: 17: 57.13$ & 9 & 90 & 15 & -20 & 20 & 20 & 20 & 94 & 48 & 302 & 38 \\
\hline 139 & $01 / 02 / 1990$ & $03: 25: 16.57$ & 8 & 95 & 10 & -20 & 20 & 20 & 20 & 105 & 48 & 304 & 41 \\
\hline 140 & $01 / 02 / 1990$ & 03:36:09.93 & 9 & 90 & 15 & -20 & 20 & 20 & 20 & 94 & 48 & 302 & 38 \\
\hline 141 & $01 / 02 / 1990$ & $04: 43: 30.58$ & 9 & 90 & 15 & -20 & 20 & 20 & 20 & 94 & 48 & 302 & 38 \\
\hline 144 & $01 / 02 / 1990$ & 05:46:02.16 & 10 & 120 & 15 & -20 & 10 & 15 & 10 & 124 & 48 & 332 & 38 \\
\hline 145 & $01 / 02 / 1990$ & $06: 12: 50.19$ & 9 & 90 & 15 & -20 & 20 & 20 & 20 & 94 & 48 & 302 & 38 \\
\hline 146 & $01 / 02 / 1990$ & 06:54:18.82 & 9 & 115 & 10 & -20 & 10 & 20 & 10 & 125 & 48 & 324 & 41 \\
\hline 147 & $01 / 02 / 1990$ & $07: 46: 38.53$ & 7 & 190 & 85 & -140 & 20 & 15 & 10 & 61 & 31 & 316 & 23 \\
\hline 148 & $01 / 02 / 1990$ & 08:56:54.99 & 8 & 65 & 30 & -70 & 10 & 8 & 10 & 108 & 71 & 320 & 16 \\
\hline 149 & $01 / 02 / 1990$ & 18:44:58.38 & 11 & 120 & 15 & -20 & 8 & 20 & 10 & 124 & 48 & 332 & 38 \\
\hline 150 & $02 / 02 / 1990$ & $02: 48: 35.13$ & 11 & 340 & 75 & -160 & 15 & 3 & 0 & 203 & 25 & 112 & 3 \\
\hline
\end{tabular}


Table II. (continued).

Paxis

Taxis

\begin{tabular}{|c|c|c|c|c|c|c|c|c|c|c|c|c|c|}
\hline $\mathbf{N}^{\circ}$ & Date & Orig. Time & $\mathbf{N}_{\mathrm{p}}$ & Strike & Dip & Rake & $\Delta$ Str & $\Delta$ Dip & $\Delta$ Rake & Azm & Plunge & Azm & Plunge \\
\hline 151 & 02/02/1990 & 05:41:31.89 & 9 & 55 & 20 & -100 & 10 & 8 & 10 & 162 & 65 & 333 & 25 \\
\hline 155 & 03/02/1990 & $23: 25: 23.83$ & 8 & 115 & 5 & -30 & 20 & 20 & 15 & 140 & 47 & 329 & 42 \\
\hline 158 & 28/02/1990 & 22:09:46.68 & 11 & 140 & 25 & -60 & 10 & 8 & 10 & 176 & 64 & 28 & 22 \\
\hline 161 & 10/06/1990 & 03:35:05.70 & 10 & 105 & 45 & -40 & 10 & 13 & 20 & 87 & 55 & 342 & 10 \\
\hline 163 & 29/09/1990 & 20:39:35.05 & 9 & 0 & 50 & -160 & 3 & 3 & 0 & 210 & 40 & 314 & 16 \\
\hline 164 & 29/09/1990 & 21:39:11.39 & 9 & 20 & 50 & -40 & 18 & 5 & 10 & 355 & 53 & 257 & 6 \\
\hline 166 & 26/11/1990 & 03:27:20.77 & 7 & 155 & 20 & -140 & 8 & 8 & 15 & 316 & 55 & 104 & 31 \\
\hline 167 & $10 / 12 / 1990$ & 04:15:15.48 & 7 & 85 & 45 & -60 & 13 & 5 & 15 & 74 & 69 & 334 & 4 \\
\hline 169 & $10 / 03 / 1991$ & $10: 35: 10.98$ & 9 & 160 & 25 & -90 & 20 & 5 & 10 & 250 & 70 & 70 & 20 \\
\hline 175 & 19/04/1992 & 02:35:55.35 & 9 & 200 & 35 & -100 & 20 & 10 & 20 & 327 & 78 & 117 & 10 \\
\hline 176 & 20/04/1992 & 05:19:12.00 & 8 & 5 & 70 & -110 & 15 & 13 & 20 & 246 & 60 & 110 & 22 \\
\hline 178 & $16 / 05 / 1992$ & $02: 52: 56.20$ & 9 & 95 & 25 & -50 & 8 & 5 & 5 & 118 & 60 & 335 & 24 \\
\hline 179 & 23/05/1992 & 01:04:56.21 & 10 & 240 & 70 & -40 & 10 & 13 & 10 & 197 & 42 & 297 & 11 \\
\hline 180 & 08/07/1992 & $05: 35: 54.28$ & 8 & 60 & 75 & 0 & 5 & 5 & 5 & 16 & 11 & 284 & 11 \\
\hline 182 & 03/08/1992 & $04: 37: 14.52$ & 9 & 195 & 80 & -170 & 5 & 10 & 20 & 59 & 14 & 149 & 0 \\
\hline 183 & 10/08/1992 & $22: 12: 19.42$ & 8 & 75 & 45 & -90 & 18 & 8 & 20 & 241 & 90 & 345 & 0 \\
\hline 185 & 11/09/1992 & $12: 05: 31.43$ & 10 & 10 & 70 & -120 & 10 & 15 & 20 & 242 & 55 & 122 & 19 \\
\hline 187 & 11/09/1992 & 21:30:43.36 & 10 & 130 & 30 & -160 & 5 & 5 & 0 & 322 & 47 & 90 & 29 \\
\hline 188 & 11/09/1992 & 21:34:12.69 & 9 & 15 & 75 & -120 & 3 & 5 & 20 & 251 & 51 & 128 & 24 \\
\hline 189 & 11/09/1992 & $21: 36: 46.91$ & 8 & 140 & 15 & -150 & 10 & 10 & 10 & 305 & 51 & 99 & 36 \\
\hline 190 & 11/09/1992 & 21:40:37.08 & 8 & 75 & 85 & -30 & 10 & 3 & 10 & 27 & 24 & 125 & 17 \\
\hline 191 & 11/09/1992 & $21: 44: 12.83$ & 8 & 25 & 75 & -110 & 10 & 8 & 20 & 270 & 56 & 131 & 27 \\
\hline 193 & 11/09/1992 & 21:59:09.57 & 8 & 165 & 65 & -180 & 5 & 10 & 0 & 27 & 17 & 123 & 17 \\
\hline 194 & 11/09/1992 & 22:00:38.98 & 10 & 250 & 85 & 30 & 8 & 5 & 5 & 20 & 17 & 118 & 24 \\
\hline 195 & 11/09/1992 & 22:01:23.17 & 8 & 195 & 20 & -60 & 18 & 10 & 10 & 238 & 61 & 82 & 27 \\
\hline 196 & 11/09/1992 & $22: 25: 24.05$ & 8 & 20 & 75 & -110 & 13 & 8 & 20 & 265 & 56 & 126 & 27 \\
\hline 197 & 11/09/1992 & 22:53:51.68 & 7 & 65 & 85 & -30 & 10 & 5 & 5 & 17 & 24 & 115 & 17 \\
\hline 200 & 12/09/1992 & 00:02:07.72 & 8 & 190 & 20 & -60 & 18 & 10 & 10 & 233 & 61 & 77 & 27 \\
\hline 201 & 12/09/1992 & 00:09:17.40 & 7 & 15 & 75 & -50 & 13 & 8 & 5 & 325 & 45 & 76 & 20 \\
\hline 205 & 12/09/1992 & 03:29:06.56 & 8 & 20 & 75 & -110 & 10 & 5 & 20 & 265 & 56 & 126 & 27 \\
\hline 206 & 12/09/1992 & 04:27:17.23 & 7 & 195 & 25 & -60 & 18 & 10 & 10 & 231 & 64 & 83 & 22 \\
\hline 207 & 12/09/1992 & $05: 37: 42.28$ & 8 & 190 & 20 & -60 & 18 & 13 & 20 & 233 & 61 & 77 & 27 \\
\hline 208 & $12 / 09 / 1992$ & $05: 38: 21.74$ & 8 & 190 & 20 & -60 & 18 & 10 & 10 & 233 & 61 & 77 & 27 \\
\hline
\end{tabular}


Table II. (continued).

\begin{tabular}{|c|c|c|c|c|c|c|c|c|c|c|c|c|c|}
\hline \multirow[b]{2}{*}{$\mathbf{N}^{\circ}$} & \multirow[b]{2}{*}{ Date } & \multirow[b]{2}{*}{ Orig. Time } & \multirow[b]{2}{*}{$\mathbf{N}_{\mathrm{p}}$} & \multirow[b]{2}{*}{ Strike } & \multirow[b]{2}{*}{ Dip } & \multirow[b]{2}{*}{ Rake } & \multirow[b]{2}{*}{$\Delta$ Str } & \multirow[b]{2}{*}{$\Delta$ Dip } & \multirow[b]{2}{*}{$\Delta$ Rake } & \multicolumn{2}{|c|}{ Paxis } & \multicolumn{2}{|c|}{ Taxis } \\
\hline & & & & & & & & & & Azm & Plunge & Azm & Plunge \\
\hline 215 & 18/09/1992 & $03: 56: 35.45$ & 7 & 175 & 70 & -120 & 15 & 5 & 5 & 47 & 55 & 287 & 19 \\
\hline 216 & 18/09/1992 & $04: 48: 24.98$ & 8 & 185 & 70 & -120 & 15 & 13 & 10 & 57 & 55 & 297 & 19 \\
\hline 218 & 29/10/1992 & $21: 16: 32.60$ & 9 & 230 & 15 & 160 & 8 & 18 & 10 & 82 & 38 & 234 & 48 \\
\hline 221 & 07/12/1992 & 04:06:00.46 & 7 & 345 & 75 & -160 & 20 & 15 & 10 & 208 & 25 & 117 & 3 \\
\hline 224 & $10 / 04 / 1993$ & $20: 50: 10.97$ & 10 & 75 & 60 & -70 & 10 & 8 & 20 & 26 & 68 & 151 & 13 \\
\hline 225 & $23 / 04 / 1993$ & 02:46:32.05 & 8 & 50 & 15 & -90 & 20 & 10 & 20 & 140 & 60 & 320 & 30 \\
\hline 227 & $02 / 06 / 1993$ & $08: 26: 14.41$ & 9 & 100 & 35 & -60 & 10 & 8 & 10 & 115 & 69 & 349 & 13 \\
\hline 229 & $30 / 06 / 1993$ & 21:20:27.01 & 9 & 300 & 80 & -150 & 15 & 15 & 10 & 166 & 28 & 69 & 13 \\
\hline 233 & 01/07/1993 & 19:01:49.32 & 10 & 290 & 85 & -140 & 10 & 8 & 0 & 161 & 31 & 56 & 23 \\
\hline 236 & 01/07/1993 & $20: 34: 56.45$ & 7 & 315 & 75 & -140 & 10 & 10 & 5 & 181 & 38 & 79 & 15 \\
\hline 237 & 01/07/1993 & 21:06:47.01 & 9 & 220 & 70 & -20 & 10 & 10 & 10 & 179 & 28 & 88 & 1 \\
\hline 238 & 01/07/1993 & 21:41:53.12 & 10 & 300 & 80 & -150 & 10 & 8 & 5 & 166 & 28 & 69 & 13 \\
\hline 239 & 01/07/1993 & $22: 55: 17.24$ & 9 & 295 & 75 & -150 & 8 & 8 & 5 & 159 & 32 & 63 & 9 \\
\hline 240 & 02/07/1993 & $00: 18: 23.24$ & 10 & 210 & 5 & 50 & 5 & 8 & 5 & 157 & 41 & 344 & 49 \\
\hline 242 & 02/07/1993 & 01:03:54.61 & 11 & 65 & 90 & 90 & 3 & 5 & 10 & 155 & 45 & 335 & 45 \\
\hline 246 & 02/07/1993 & $16: 17: 51.98$ & 9 & 320 & 5 & -20 & 10 & 20 & 20 & 335 & 47 & 164 & 43 \\
\hline 247 & 15/07/1993 & 10:18:04.47 & 9 & 75 & 75 & 60 & 8 & 3 & 10 & 188 & 24 & 311 & 51 \\
\hline 250 & $13 / 09 / 1993$ & 11:51:20.14 & 7 & 190 & 30 & -40 & 18 & 13 & 20 & 194 & 57 & 64 & 23 \\
\hline 251 & $18 / 12 / 1993$ & 06:53:57.30 & 8 & 65 & 50 & -170 & 20 & 8 & 10 & 279 & 33 & 23 & 21 \\
\hline
\end{tabular}

tainties range between 5 and 20 degrees, with the great majority of cases between 10 and 15 degrees. The initial inversion consisted of a search of the entire range of possible stress orientations on a 10-degree grid using the approximate method. The regions of possible solutions suggested by the approximate method were then searched more thoroughly using the exact method on a 5-degree grid. The ratio $\mathrm{R}$ was searched at intervals of 0.1 .

First, the entire dataset of focal mechanisms was inverted. The inversion led us to find an approximately NE-SW vertical $\sigma_{l}$ and horizontal $\sigma_{2}$ and $\sigma_{3}$ (fig. 3a and table III, dataset a). The stress ratio value $(R=0.7)$ indicates an almost uniaxial deviatoric compression. The average misfit for this inversion is 6.2. This value suggests that this dataset may be slightly contaminated by heterogeneity.

In order to test the stability of the results in this region, we also performed a second stress inversion by following both the criteria of homogeneity in the dataset (i.e. fault plane solution categories and $\mathrm{P}$ - and T-axes orientation) and the structural-geological setting of the area. Therefore, the dataset of focal mechanisms was divided into two subsets (fig. 3b,c and table III, datasets $b$ and $c$ ) as a function of space based on the pattern of seismicity distribution along the surface geologic structure NE-SW trending of Cittanova normal fault system.

For each subset several tests were performed using different grid steps and a large variety of starting values for the stress parame- 
ters in order to check the reliability of the results. For the first investigated sub volume (along the CF, dataset $\mathrm{b}$ in table III and fig. 3b), we inverted 83 focal mechanisms obtaining an average misfit of 5.6. The previously described indication of a NW-SE extension in this part of the belt is confirmed. The inversion of the remaining 37 events, located westward and northwestward of CF, gives again a horizontal $\sigma_{3}$, oriented approximately WNW-ESE, and a ver-
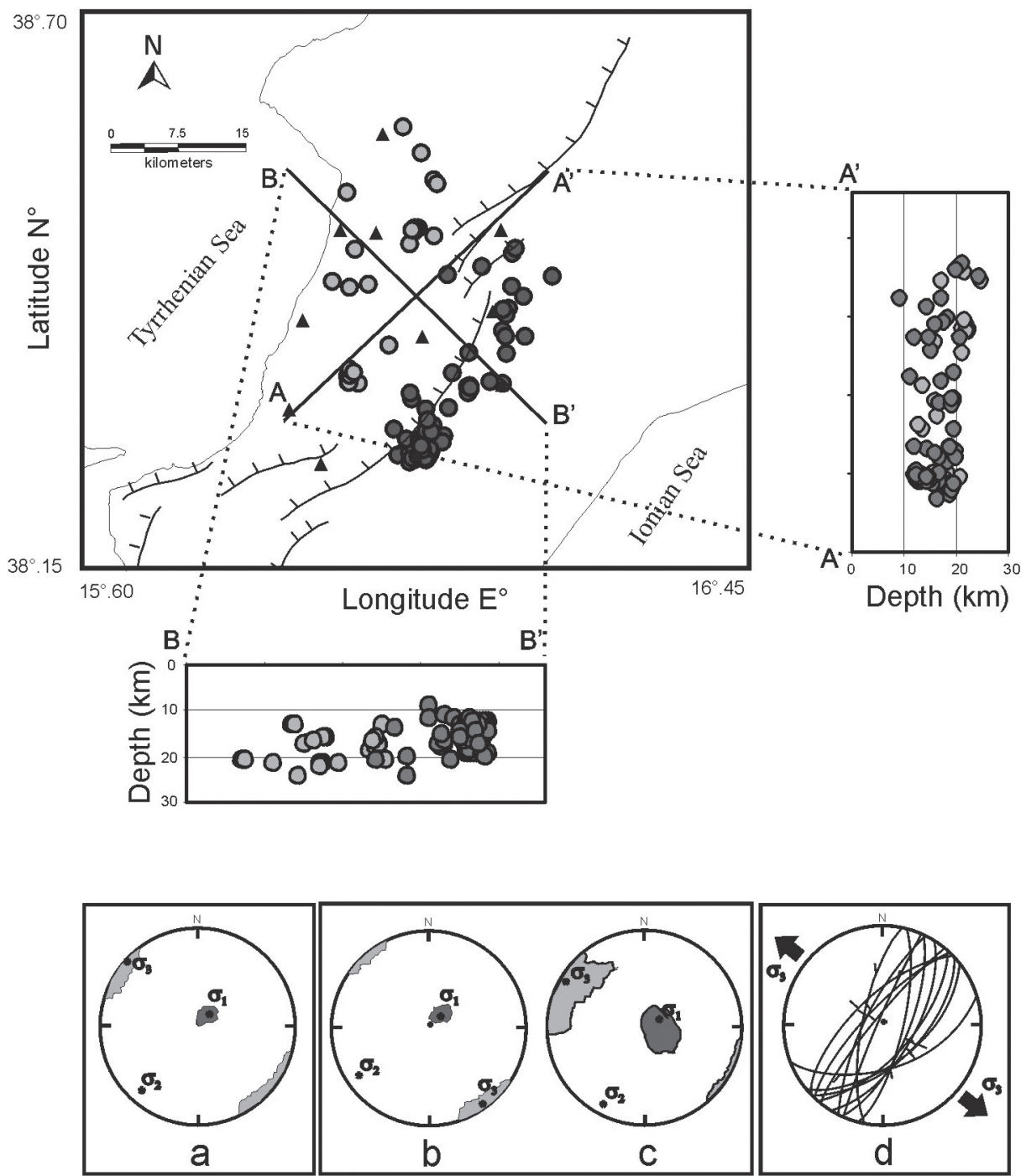

Fig. 3a-d. Map and SW-NE (A-A') and NW-SE (B-B') cross sections of the earthquakes selected for stress tensor inversion. Lower-hemisphere projection of the orientations (stars) of the principal stress axes obtained by inverting the whole dataset (a) and the two different subsets (b) and (c). Gray areas indicate the $90 \%$ confidence limits of $\sigma_{1}$ and $\sigma_{3}$ orientations. d) Diagram (Schmidt net, lower hemisphere) showing fault planes and slickensides measured along cataclastic belts of Cittanova fault. Large arrows indicate azimuth of the mean extension direction derived from a qualitative and quantitative analysis of the slickenside data sets (Tortorici et al., 1995). 
tical $\sigma_{l}$ (dataset $\mathrm{c}$ in table III and fig. 3c). The average misfit is $6.0^{\circ}$ which is the limit for ac- ceptable results and may indicate that this dataset is not completely homogeneous.

Table III. Results from Stress Inversion Runs. N, F and R are, respectively, the number of events, the average misfit corresponding to the stress solution found and the measure of relative stress magnitude. Deviatoric principal stress axes, $\sigma_{1}, \sigma_{2}, \sigma_{3}$, are the compressional, intermediate and extensional deviatoric axes, respectively. They are specified by plunges measured from horizontal and azimuth measured clockwise from north. For the corresponding dataset, see the text.

\begin{tabular}{|c|c|c|c|c|c|c|c|c|c|}
\hline \multirow[t]{2}{*}{ Dataset } & \multirow[t]{2}{*}{$\mathbf{N}$} & \multirow[t]{2}{*}{$\mathbf{F}\left({ }^{\circ}\right)$} & \multicolumn{2}{|c|}{$\sigma_{1}$} & \multicolumn{2}{|c|}{$\sigma_{2}$} & \multicolumn{2}{|c|}{$\sigma_{3}$} & \multirow[t]{2}{*}{$\mathbf{R}$} \\
\hline & & & $\mathbf{P I}\left(^{\circ}\right)$ & $\mathbf{A z}\left(^{\circ}\right)$ & $\mathbf{P I}\left({ }^{\circ}\right)$ & $\mathbf{A z}\left(^{(0}\right)$ & $\mathbf{P I}\left({ }^{\circ}\right)$ & $\mathbf{A z}\left(^{\circ}\right)$ & \\
\hline $\mathrm{a}$ & 120 & 6.2 & 76 & 45 & 14 & 222 & 1 & 312 & 0.7 \\
\hline $\mathrm{b}$ & 83 & 5.6 & 76 & 45 & 14 & 236 & 3 & 145 & 0.8 \\
\hline $\mathrm{c}$ & 37 & 6.0 & 76 & 65 & 11 & 209 & 8 & 300 & 0.8 \\
\hline
\end{tabular}

\subsection{Evaluation of $Q_{P}$}

The method here used for $Q_{P}$ estimate is based on the time domain formulation deriving from the classic rise time (or pulse-broadening) method (e.g. Gladwin and Stacey, 1974; Wu and Lees, 1996). It is based on the measure of the first $\mathrm{P}$ pulse width $\tau$ in time domain, under the assumption that the quality factor $Q$ is frequency-independent. The pulse width $\tau$, for velocity records, can be defined as the time difference from the onset to the first zero crossing (fig. 4). For point-like impulsive sources it is expressed by the linear relation:

$$
\tau=\tau_{0}+C T / Q_{p}
$$

where $\tau_{0}$ is the original pulse width at the source, $T$ is the travel time, $Q_{P}$ is the quality factor of $\mathrm{P}$-waves and $\mathrm{C}$ is a constant which is equal to 0.5 for a constant $Q$ attenuation operator (Kjartansson, 1979). The linear relation between $\tau$ and $T / Q_{P}$ predicted by equation (3.1) holds for point-like sources. In fact, the only limiting assumption of this method is that it neglects the directivity effect of the seismic radiation generated by a finite dimension seismic source (Zollo and de Lorenzo, 2001).

The rise time method is expected to give more reliable estimates of the intrinsic attenuation than spectral techniques for several reasons. First, the rise time is not affected by non- objective criteria of phase windowing; second, since only a very limited portion of the seismogram is used, the effects of secondary arrivals due to waves diffracted by heterogeneities in the medium and site effects are minimized. Moreover, since the rise time method does not use wave amplitudes, correction for the instrumental response is not needed provided that the latter is flat in the dominant frequency range of the earthquakes (e.g. Zollo and de Lorenzo, 2001). However, to take full advantage of this method it is essential that the shape of the pulse to be used is not greatly distorted by the seismogram. Therefore, the most reliable results will be obtained using signals recorded with a high signal-to-noise ratio. Following this criterion, from the entire dataset shown in fig. 1 and table I, we selected 110 earthquakes with magnitude ranging between 1.0 and 3.0 and depth ranging between 10 and $25 \mathrm{~km}$. A total of 607 waveforms with clear P-wave onset, high signal-to-noise ratio and not affected by multipathing during the first half-cycle of the wave, were considered suitable for the application of the rise time method. We did not perform the correction for the instrumental response because the filtering operated in the deconvolution can generate artificial signals (Mulargia and Geller, 2003 and references therein). Moreover, since the frequency content of first pulses is always contained in the range where the response of the instruments is flat, rise times should be un- 


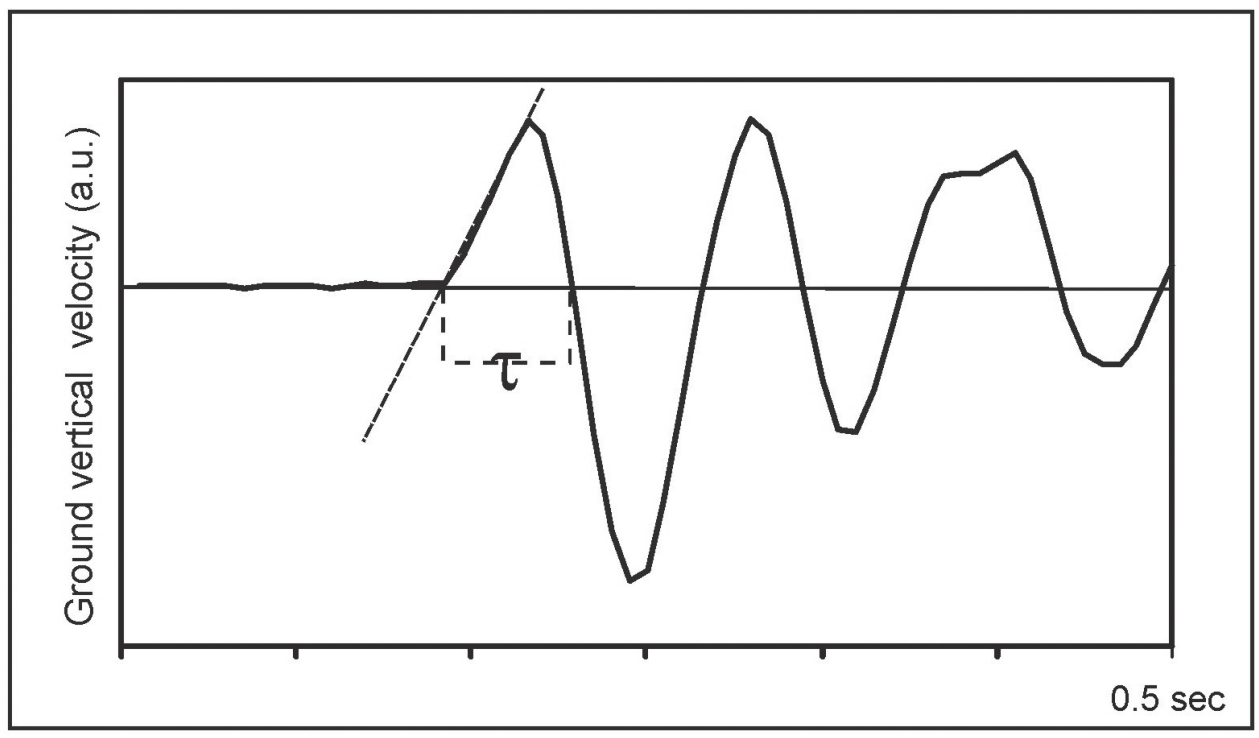

Fig. 4. Schematic picture of rise time as measured on a velocity seismogram.

affected by instrumental effects. The rise time of P-waves was measured on each vertical seismogram using a semi-automatic procedure which computes the time interval between the onset of the P-wave and its first zero crossing.

We first plotted all $\tau$ values vs. travel time $(T)$ considering the minimum $1 \mathrm{D}$ velocity model proposed by Raffaele et al. (2006). A first observation consists of the positive trend of rise times vs. the travelled distances, even though some scatter in the data is observed (fig. 5). The dispersion of the data around the line of best fit may be due either to spatial variations in the attenuation properties of the ray sampled volume and/or to directivity source effects. Under the simplified assumption of a non-directive source we obtained an average estimate of $Q_{P}$ as $84 \pm 8$. Then, in order to estimate the intrinsic attenuation at each station site and to evidence possible lateral variations in the anelastic properties of the medium, we plotted $\tau$ values vs. $T$ for each station. Table IV reports the inferred estimates of $Q_{P}$ at 10 stations (we did not include GTSM station because of the low number of data available), together with the relative standard deviation. The $Q_{P}$ values obtained at most of the stations are, within the error, very close to the average $Q_{P}$ obtained for the whole area. However, lower $Q_{P}$ values are obtained at stations DLV and ZMR and higher $Q_{P}$ are found at stations CCL and STC.

Finally, we plotted $\tau$ values vs. $T$ for each event and computed the best fit straight line. Some of these fits are reported, as examples, in fig. 6. The events for which the slope of the

Table IV. $Q_{P}$ values and relative errors at each seismic station.

\begin{tabular}{cc}
\hline \hline Station Name & $\mathbf{Q}_{\mathbf{P} \pm \boldsymbol{\sigma}}$ \\
\hline CCL & $100 \pm 24$ \\
CGT & $56 \pm 16$ \\
CLT & $86 \pm 30$ \\
CSB & $77 \pm 19$ \\
CST & $54 \pm 15$ \\
DLV & $38 \pm 9$ \\
MTR & $78 \pm 20$ \\
PDL & $68 \pm 17$ \\
STC & $149 \pm 53$ \\
ZMR & $36 \pm 4$ \\
\hline
\end{tabular}




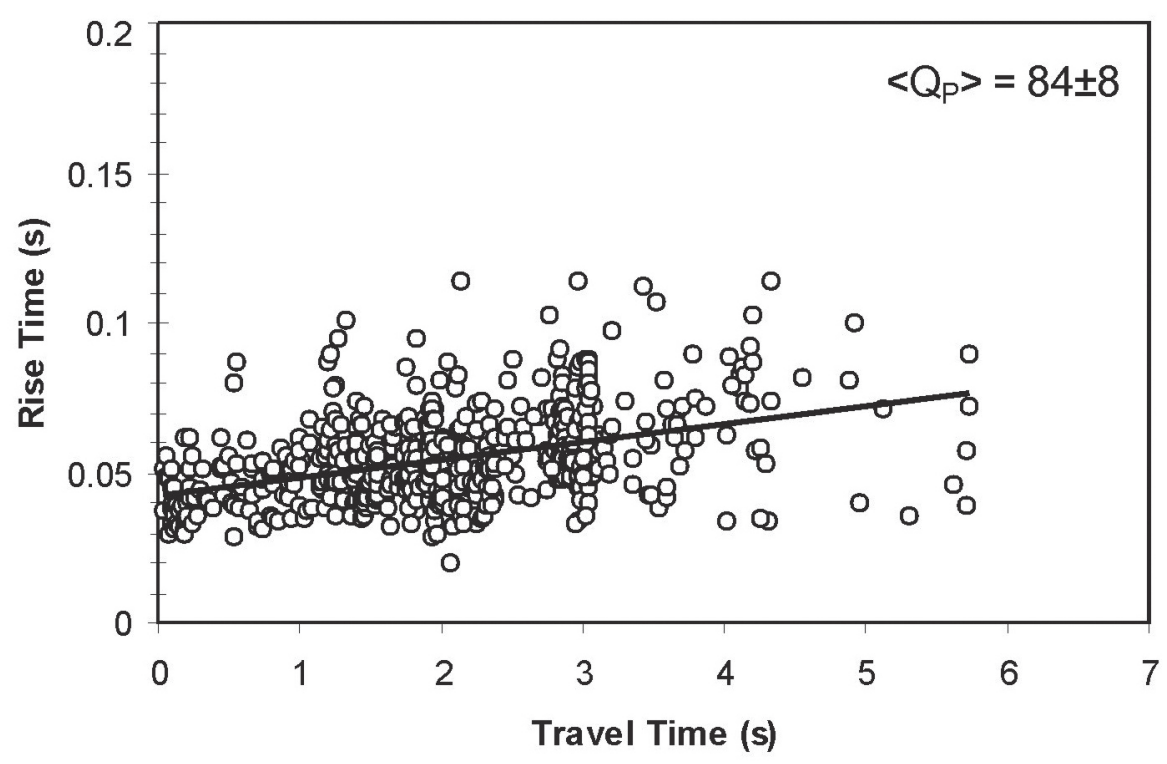

Fig. 5. Rise time plotted as a function of travel time. The best fit interpolating straight line is also shown.

Ev. 20.11.1985 07:24 h= $18.5 \mathrm{~km} \mathrm{M}=1.7$

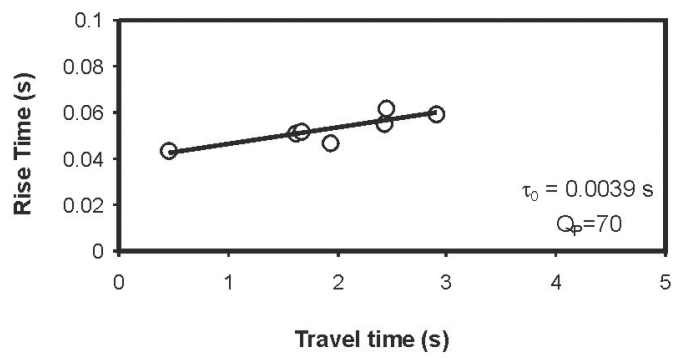

Ev. $13.12 .198918 .35 \mathrm{~h}=11.1 \mathrm{~km} \mathrm{M}_{\mathrm{L}}=1.9$

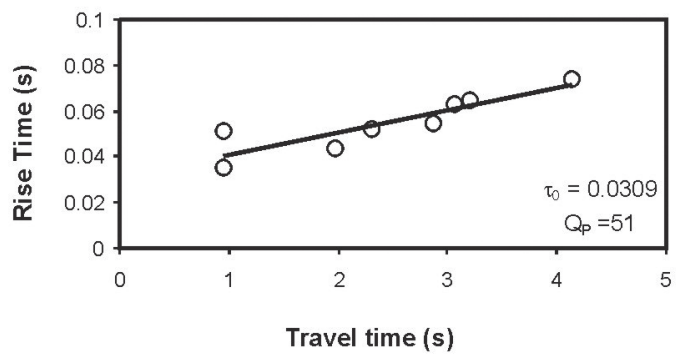

Ev. 08.03.1986 $11.30 \mathrm{~h}=18.1 \mathrm{~km} \mathrm{M}=1.3$

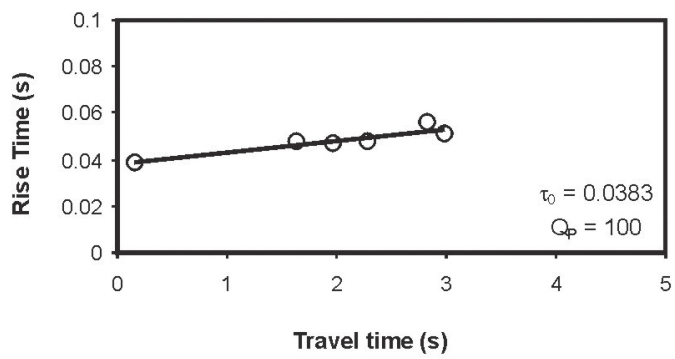

Ev. 01.02.1990 10.31 h= 21.7 km $\mathrm{M}_{\mathrm{L}}=2.1$

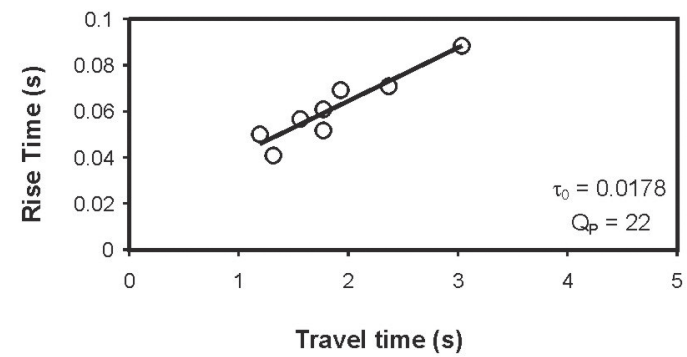

Fig. 6. Rise time vs. travel time for some events considered in this study. The best fitting straight line is also shown. 
straight line described by equation (3.1) was negative have no physical significance and were discarded. A total of 89 events were considered. We obtained, for each event, a $Q_{P}$ value ranging between 14 and 400 and an average $Q_{P}$ $=87$, which is very close to the average value obtained from the linear regression of the whole dataset.

\subsection{Source parameters estimate}

For a circular crack, the source rise time, which represents the time duration of the slipping on the fault, is related to the source radius $L$ and the rupture velocity (Boatwright, 1980). We used the source rise times $\tau_{0}$ calculated for each of the 89 events to estimate the source dimension $L$ by using the following relationship:

$$
\tau_{0}=L / V_{\mathrm{r}}
$$

where $V_{r}$ is the average rupture velocity here assumed equal to $0.9 V_{S}$, with $V_{S}$ the average $\mathrm{S}$-wave velocity. Considering the average $V_{S}$ $=3.6 \mathrm{~km} / \mathrm{s}$ (Raffaele et al., 2006), the used $V_{r}$ is $3.24 \mathrm{~km} / \mathrm{s}$. We obtained source radii varying from a few meters to $200 \mathrm{~m}$ (table V).

For each event the seismic moment $M_{0}$ was calculated from the estimated local magnitude $M_{L}$ (table V) by using the general empirical relationship by Bakun and Lindth (1977):

$$
\log M_{0}=1.2 M_{L}+17
$$

The estimated $M_{0}$ values were plotted vs. source dimension in fig. 7a. The lines of constant stress drop $\Delta \sigma$ were obtained by using the scaling relationship for circular earthquake sources (Keilis-Borok, 1959):

$$
\Delta \sigma=\frac{7 M_{0}}{16 L^{3}}
$$

The stress drop of most of the events is low and concentrated between 0.1 and 10 bars, with an average value around 5 bars. Only few events have higher stress drop, up to 100 bars (figs. $7 \mathrm{a}, \mathrm{b}$ and 8 ; table V). It must be stressed that according to equation (3.2), source radius esti-
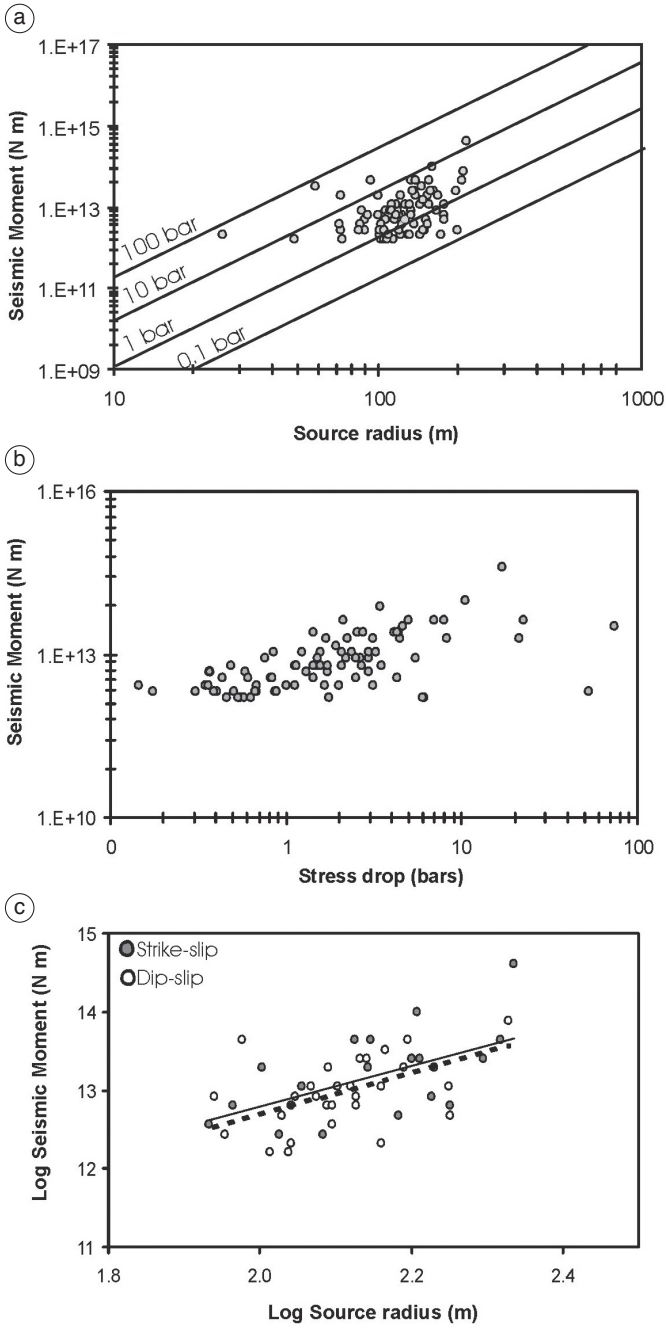

Fig. 7a-c. a) Seismic moment vs. source dimensions. The lines of constant stress drop are also reported. b) Seismic moment vs. stress drop. c) Scaling relations seismic moment-source radius for dip-slip (black line) and strike-slip (black dotted line) source mechanisms.

mates, and therefore stress drop, are rupture velocity-dependent (e.g. Zollo and de Lorenzo, 2001). The average rupture velocity of small magnitude events is usually poorly known. Theoretical and laboratory studies (Madariaga, 1976) indicate that $V_{r}$ varies between $0.6 V_{S}$ and 
Table V. Source parameters of the analysed events.

\begin{tabular}{|c|c|c|c|c|c|c|}
\hline $\mathbf{N}^{\circ}$ & Date & Orig. Time & $\mathbf{M}_{\mathbf{L}}$ & $\mathbf{L}(\mathbf{m})$ & $\mathbf{M}_{0}(\mathbf{N} \mathbf{m})$ & $\Delta \sigma($ bars $)$ \\
\hline 4 & $28 / 04 / 1985$ & $22: 41: 03.14$ & 1.4 & 178 & $4.79 \mathrm{E}+12$ & 0.37 \\
\hline 8 & $24 / 08 / 1985$ & $17: 56: 37.65$ & 1 & 73 & $1.58 \mathrm{E}+12$ & 1.77 \\
\hline 10 & $26 / 10 / 1985$ & $16: 52: 03.04$ & 1.2 & 151 & $2.75 \mathrm{E}+12$ & 0.35 \\
\hline 11 & $27 / 10 / 1985$ & $06: 44: 54.88$ & 1.5 & 101 & $6.31 \mathrm{E}+12$ & 2.72 \\
\hline 12 & $28 / 10 / 1985$ & $18: 41: 47.79$ & 2.2 & 134 & $4.37 \mathrm{E}+13$ & 8 \\
\hline 14 & $20 / 11 / 1985$ & $17: 07: 22.24$ & 1.7 & 127 & $1.10 \mathrm{E}+13$ & 2.35 \\
\hline 18 & $27 / 12 / 1985$ & $18: 00: 10.63$ & 2 & 163 & $2.51 \mathrm{E}+13$ & 2.55 \\
\hline 20 & $27 / 12 / 1985$ & $20: 07: 31.85$ & 1.2 & 90 & $2.75 \mathrm{E}+12$ & 1.65 \\
\hline 21 & $27 / 12 / 1985$ & $20: 07: 49.31$ & 1.4 & 152 & $4.79 \mathrm{E}+12$ & 0.59 \\
\hline 22 & $28 / 12 / 1985$ & $14: 52: 24.25$ & 1.9 & 123 & $1.91 \mathrm{E}+13$ & 4.48 \\
\hline 23 & $29 / 12 / 1985$ & 19:56:02.99 & 1 & 115 & $1.58 \mathrm{E}+12$ & 0.46 \\
\hline 24 & $04 / 01 / 1986$ & $02: 37: 06.54$ & 1.6 & 107 & $8.32 \mathrm{E}+12$ & 2.96 \\
\hline 28 & $23 / 02 / 1986$ & $15: 33: 29.42$ & 1.5 & 110 & $6.31 \mathrm{E}+12$ & 2.07 \\
\hline 30 & $23 / 02 / 1986$ & $15: 42: 31.76$ & 1.2 & 202 & $2.75 \mathrm{E}+12$ & 0.15 \\
\hline 31 & $24 / 02 / 1986$ & $10: 09: 56.51$ & 1.5 & 123 & $6.31 \mathrm{E}+12$ & 1.5 \\
\hline 32 & 08/03/1986 & $03: 11: 28.64$ & 1.3 & 125 & $3.63 \mathrm{E}+12$ & 0.82 \\
\hline 33 & 08/03/1986 & $17: 39: 09.65$ & 1 & 103 & $1.58 \mathrm{E}+12$ & 0.63 \\
\hline 35 & $12 / 03 / 1986$ & $03: 34: 13.93$ & 2.2 & 95 & $4.37 \mathrm{E}+13$ & 22.54 \\
\hline 36 & $12 / 03 / 1986$ & $03: 36: 04.43$ & 2.2 & 157 & $4.37 \mathrm{E}+13$ & 4.96 \\
\hline 38 & $12 / 03 / 1986$ & 03:54:00.19 & 1.9 & 155 & $1.91 \mathrm{E}+13$ & 2.23 \\
\hline 39 & $12 / 03 / 1986$ & 05:00:06.66 & 1 & 108 & $1.58 \mathrm{E}+12$ & 0.54 \\
\hline 41 & $12 / 03 / 1986$ & $05: 53: 25.81$ & 2.1 & 147 & $3.31 \mathrm{E}+13$ & 4.61 \\
\hline 42 & $12 / 03 / 1986$ & $06: 58: 59.54$ & 1.7 & 178 & $1.10 \mathrm{E}+13$ & 0.85 \\
\hline 44 & $12 / 03 / 1986$ & $15: 21: 38.95$ & 1.4 & 107 & $4.79 \mathrm{E}+12$ & 1.71 \\
\hline 45 & $12 / 03 / 1986$ & 18:55:09.54 & 1.5 & 124 & $6.31 \mathrm{E}+12$ & 1.43 \\
\hline 49 & $17 / 06 / 1986$ & $18: 46: 00.44$ & 2 & 159 & $2.51 \mathrm{E}+13$ & 2.74 \\
\hline 50 & $17 / 07 / 1986$ & $07: 30: 31.86$ & 1.2 & 73 & $2.75 \mathrm{E}+12$ & 3.15 \\
\hline 55 & $24 / 08 / 1986$ & $02: 38: 37.50$ & 1.1 & 131 & $2.09 \mathrm{E}+12$ & 0.41 \\
\hline 56 & $24 / 08 / 1986$ & $02: 52: 31.69$ & 1.1 & 102 & $2.09 \mathrm{E}+12$ & 0.86 \\
\hline 57 & $24 / 08 / 1986$ & $20: 13: 25.54$ & 1.5 & 120 & $6.31 \mathrm{E}+12$ & 1.59 \\
\hline 58 & $26 / 08 / 1986$ & $06: 54: 18.47$ & 1.6 & 119 & $8.32 \mathrm{E}+12$ & 2.18 \\
\hline 59 & $26 / 08 / 1986$ & $14: 15: 29.71$ & 1.1 & 102 & $2.09 \mathrm{E}+12$ & 0.87 \\
\hline 60 & $23 / 09 / 1986$ & $20: 20: 35.27$ & 1.1 & 145 & $2.09 \mathrm{E}+12$ & 0.3 \\
\hline
\end{tabular}


Table V. (continued).

\begin{tabular}{|c|c|c|c|c|c|c|}
\hline $\mathbf{N}^{\circ}$ & Date & Orig. Time & $\mathbf{M}_{\mathbf{L}}$ & $\mathbf{L}(\mathbf{m})$ & $\mathbf{M}_{0}(\mathbf{N} \mathbf{m})$ & $\Delta \sigma$ (bars) \\
\hline 61 & $19 / 10 / 1986$ & 04:09:52.22 & 1.3 & 104 & $3.63 \mathrm{E}+12$ & 1.43 \\
\hline 64 & $27 / 11 / 1986$ & $19: 26: 51.38$ & 1.1 & 101 & $2.09 \mathrm{E}+12$ & 0.88 \\
\hline 67 & $16 / 12 / 1986$ & 02:13:16.01 & 1.7 & 117 & $1.10 \mathrm{E}+13$ & 2.99 \\
\hline 72 & 03/05/1987 & 04:07:40.12 & 1.7 & 145 & $1.10 \mathrm{E}+13$ & 1.58 \\
\hline 73 & $12 / 05 / 1987$ & $11: 34: 11.23$ & 1.2 & 102 & $2.75 \mathrm{E}+12$ & 1.12 \\
\hline 74 & $12 / 05 / 1987$ & $11: 36: 06.70$ & 1.4 & 117 & $4.79 \mathrm{E}+12$ & 1.31 \\
\hline 77 & $12 / 05 / 1987$ & $12: 14: 59.85$ & 1.7 & 114 & $1.10 \mathrm{E}+13$ & 3.28 \\
\hline 78 & $12 / 05 / 1987$ & $12: 17: 39.33$ & 1.5 & 178 & $6.31 \mathrm{E}+12$ & 0.49 \\
\hline 80 & $12 / 05 / 1987$ & $12: 28: 38.11$ & 2 & 136 & $2.51 \mathrm{E}+13$ & 4.39 \\
\hline 84 & $14 / 06 / 1987$ & 18:24:08.19 & 2 & 138 & $2.51 \mathrm{E}+13$ & 4.15 \\
\hline 89 & $18 / 12 / 1987$ & $21: 17: 29.50$ & 1.3 & 72 & $3.63 \mathrm{E}+12$ & 4.3 \\
\hline 90 & $24 / 01 / 1988$ & 01:50:50.12 & 3 & 217 & $3.98 \mathrm{E}+14$ & 17.13 \\
\hline 91 & $24 / 01 / 1988$ & $18: 45: 36.87$ & 1.7 & 157 & $1.10 \mathrm{E}+13$ & 1.23 \\
\hline 96 & 02/05/1988 & 02:41:02.81 & 1.6 & 111 & $8.32 \mathrm{E}+12$ & 2.63 \\
\hline 101 & $21 / 07 / 1988$ & 10:18:59.62 & 1.3 & 137 & $3.63 \mathrm{E}+12$ & 0.61 \\
\hline 105 & $11 / 01 / 1989$ & $11: 36: 36.58$ & 1.1 & 122 & $2.09 \mathrm{E}+12$ & 0.5 \\
\hline 107 & 07/03/1989 & $18: 52: 03.75$ & 1 & 106 & $1.58 \mathrm{E}+12$ & 0.58 \\
\hline 108 & 09/03/1989 & $16: 41: 41.39$ & 1.5 & 135 & $6.31 \mathrm{E}+12$ & 1.13 \\
\hline 109 & $29 / 05 / 1989$ & 18:59:47.09 & 1 & 48 & $1.58 \mathrm{E}+12$ & 6.12 \\
\hline 111 & 04/06/1989 & $02: 20: 47.67$ & 1.1 & 110 & $2.09 \mathrm{E}+12$ & 0.68 \\
\hline 112 & 04/06/1989 & $22: 36: 21.41$ & 1.5 & 134 & $6.31 \mathrm{E}+12$ & 1.15 \\
\hline 113 & 04/06/1989 & 22:58:02.94 & 1 & 109 & $1.58 \mathrm{E}+12$ & 0.53 \\
\hline 114 & 04/06/1989 & $23: 56: 18.07$ & 1.2 & 150 & $2.75 \mathrm{E}+12$ & 0.36 \\
\hline 115 & 04/06/1989 & $23: 57: 03.76$ & 1.6 & 113 & $8.32 \mathrm{E}+12$ & 2.49 \\
\hline 116 & 05/06/1989 & 04:09:21.01 & 1.6 & 87 & $8.32 \mathrm{E}+12$ & 5.53 \\
\hline 117 & 06/06/1989 & $00: 06: 36.62$ & 1.1 & 133 & $2.09 \mathrm{E}+12$ & 0.39 \\
\hline 127 & $26 / 08 / 1989$ & 09:40:08.65 & 1.4 & 89 & $4.79 \mathrm{E}+12$ & 2.95 \\
\hline 128 & $13 / 12 / 1989$ & $18: 35: 49.30$ & 1.9 & 101 & $1.91 \mathrm{E}+13$ & 8.2 \\
\hline 129 & $21 / 12 / 1989$ & 09:00:32.11 & 1.5 & 117 & $6.31 \mathrm{E}+12$ & 1.72 \\
\hline 130 & $21 / 12 / 1989$ & 11:20:23.95 & 2.2 & 140 & $4.37 \mathrm{E}+13$ & 6.97 \\
\hline 131 & $21 / 12 / 1989$ & $12: 21: 35.23$ & 1.2 & 106 & $2.75 \mathrm{E}+12$ & 1.01 \\
\hline 133 & $31 / 01 / 1990$ & 21:38:04.83 & 1.8 & 148 & $1.45 \mathrm{E}+13$ & 1.93 \\
\hline
\end{tabular}


Table V. (continued).

\begin{tabular}{|c|c|c|c|c|c|c|}
\hline $\mathbf{N}^{\circ}$ & Date & Orig. Time & $\mathbf{M}_{\mathbf{L}}$ & $\mathbf{L}(\mathbf{m})$ & $\mathbf{M}_{0}(\mathbf{N} \mathbf{m})$ & $\Delta \sigma$ (bars) \\
\hline 134 & $31 / 01 / 1990$ & $21: 38: 17.50$ & 1.6 & 134 & $8.32 \mathrm{E}+12$ & 1.51 \\
\hline 136 & $01 / 02 / 1990$ & $00: 47: 27.58$ & 1.5 & 92 & $6.31 \mathrm{E}+12$ & 3.51 \\
\hline 137 & $01 / 02 / 1990$ & 01:31:18.08 & 2.1 & 58 & $3.31 \mathrm{E}+13$ & 74.52 \\
\hline 138 & $01 / 02 / 1990$ & 03:17:57.13 & 1.3 & 86 & $3.63 \mathrm{E}+12$ & 2.52 \\
\hline 139 & $01 / 02 / 1990$ & $03: 25: 16.57$ & 1.2 & 121 & $2.75 \mathrm{E}+12$ & 0.68 \\
\hline 140 & $01 / 02 / 1990$ & 03:36:09.93 & 1.9 & 139 & $1.91 \mathrm{E}+13$ & 3.11 \\
\hline 141 & $01 / 02 / 1990$ & $04: 43: 30.58$ & 1.3 & 154 & $3.63 \mathrm{E}+12$ & 0.43 \\
\hline 142 & $01 / 02 / 1990$ & $04: 53: 20.05$ & 1.1 & 174 & $2.09 \mathrm{E}+12$ & 0.17 \\
\hline 143 & $01 / 02 / 1990$ & 05:04:25.49 & 1.6 & 168 & $8.32 \mathrm{E}+12$ & 0.76 \\
\hline 145 & $01 / 02 / 1990$ & $06: 12: 50.19$ & 1.9 & 73 & $1.91 \mathrm{E}+13$ & 21.36 \\
\hline 146 & $01 / 02 / 1990$ & $06: 54: 18.82$ & 2 & 197 & $2.51 \mathrm{E}+13$ & 1.43 \\
\hline 149 & $01 / 02 / 1990$ & $18: 44: 58.38$ & 1.9 & 170 & $1.91 \mathrm{E}+13$ & 1.7 \\
\hline 151 & $02 / 02 / 1990$ & $05: 41: 31.89$ & 1.1 & 26 & $2.09 \mathrm{E}+12$ & 53.29 \\
\hline 152 & $02 / 02 / 1990$ & $20: 12: 47.40$ & 1.2 & 85 & $2.75 \mathrm{E}+12$ & 1.99 \\
\hline 157 & $11 / 02 / 1990$ & 21:29:07.67 & 2.4 & 213 & $7.59 \mathrm{E}+13$ & 3.45 \\
\hline 158 & $28 / 02 / 1990$ & $22: 09: 46.68$ & 1.3 & 124 & $3.63 \mathrm{E}+12$ & 0.83 \\
\hline 159 & $12 / 03 / 1990$ & 19:10:16.12 & 1.1 & 111 & $2.09 \mathrm{E}+12$ & 0.67 \\
\hline 160 & $16 / 05 / 1990$ & 21:33:08.97 & 1 & 49 & $1.58 \mathrm{E}+12$ & 6 \\
\hline 163 & $29 / 09 / 1990$ & $20: 39: 35.05$ & 2.2 & 208 & $4.37 \mathrm{E}+13$ & 2.12 \\
\hline 164 & $29 / 09 / 1990$ & 21:39:11.39 & 2.5 & 161 & $1.00 \mathrm{E}+14$ & 10.46 \\
\hline 168 & $31 / 01 / 1991$ & $06: 57: 55.05$ & 2 & 137 & $2.51 \mathrm{E}+13$ & 4.32 \\
\hline 169 & $10 / 03 / 1991$ & $10: 35: 10.98$ & 1.7 & 132 & $1.10 \mathrm{E}+13$ & 2.08 \\
\hline 170 & $20 / 04 / 1991$ & $03: 26: 20.14$ & 1.5 & 134 & $6.31 \mathrm{E}+12$ & 1.15 \\
\hline 246 & $02 / 07 / 1993$ & $16: 17: 51.98$ & 1.5 & 20 & $1.58 \mathrm{E}+12$ & 0.15 \\
\hline
\end{tabular}

$0.9 V_{S}$. On the basis of equation (3.2), source radii have been rescaled by assuming $V_{r}=0.6$ $V_{S}$. The resulting average stress drop is 15 bars, against the 5 bars average value obtained assuming $V_{r}=0.9 V_{S}$. Therefore, stress drop values of most of the analysed events remain low, even changing the velocity rupture.
Possible differences among the scaling relationships of source parameters for earthquakes of different faulting mechanisms were also investigated.

The plot of source radius vs. seismic moment for dip-slip and strike-slip fault mechanisms is shown in fig. $7 \mathrm{c}$. 


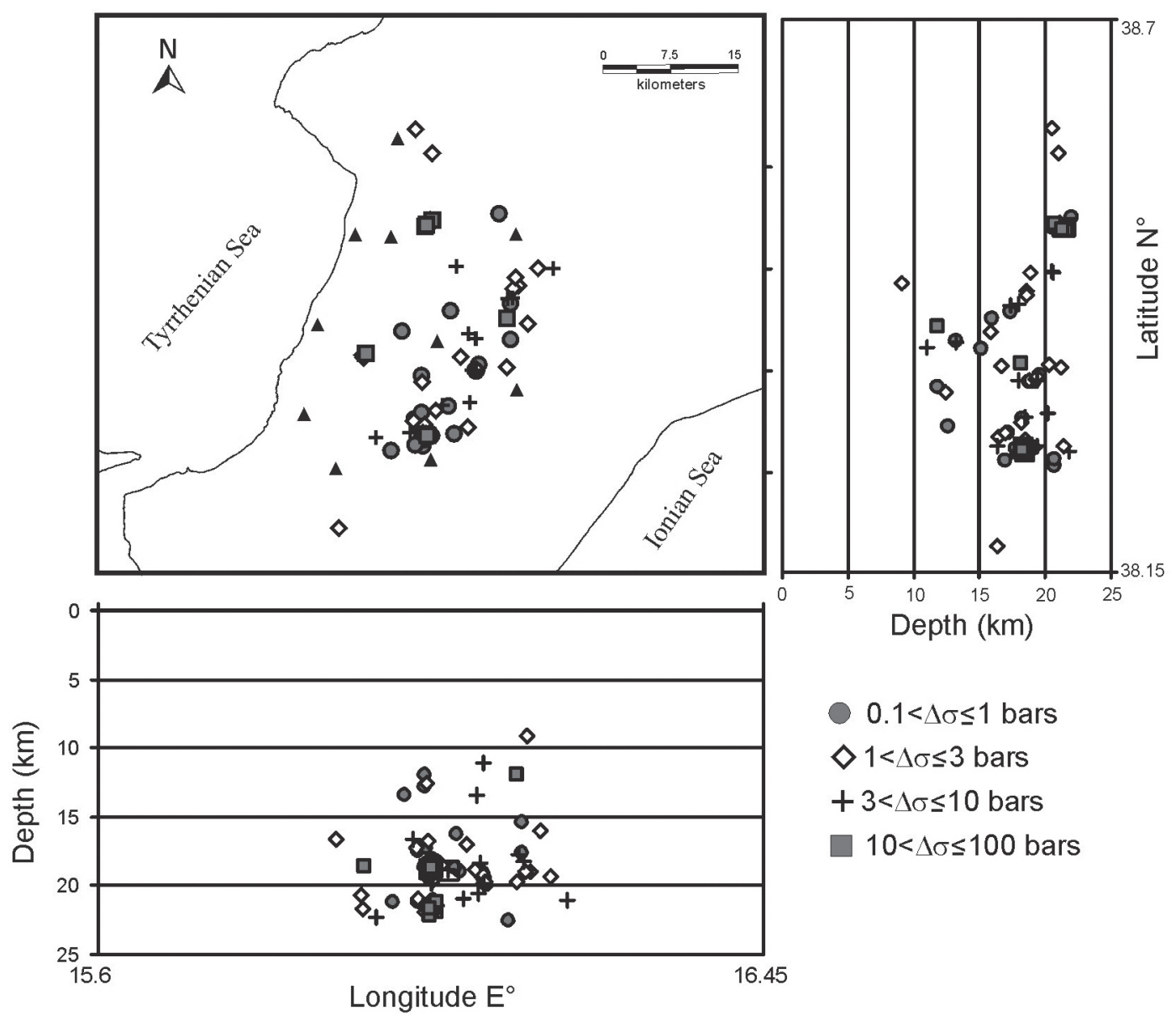

Fig. 8. Map and cross sections showing the spatial distribution of stress drop for the analysed events.

\section{Discussion and conclusions}

Data from a 9-year time span recorded by a temporary network deployed in southern Calabria from 1985 to 1994 were analyzed for earthquake focal mechanisms, stress tensor inversion, P-wave seismic attenuation and earthquake source parameters estimation. Although the seismic activity analyzed in this study may not be representative of the seismic behaviour of the whole area, we believe that the findings of this study may have some noteworthy implications for the prediction of the style of faulting of the region and for the comprehension of earthquake source physics.

Epicentral distribution of selected dataset (figs. 1 and 3) displays a clustering of events along a NE-SW trend, which corresponds to a segment of the well-documented Cittanova fault. The NW-SE and SW-NE cross sections (fig. 3) indicate that the depth of seismicity generally extends to about $25 \mathrm{~km}$, with depth mainly concentrated from 10 to $25 \mathrm{~km}$. Looking at the focal mechanisms of the events two main classes of solutions may be recognized: normal and strike-slip solutions. Normal slip solutions slightly prevail over the other. In gen- 
eral, the choice of the fault plane is problematic, except when there is a clear morphological evidence of the fault at the surface and/or the epicentral distribution is well constrained and shows a clear elongation (Patanè and Privitera, 2001). In our case, FPSs show prevalent rupture orientations occurring along ca. NE-SW directions (fig. 2), in agreement with the overall distribution of foci (fig. 3) and structural setting (fig. 1). Moreover, the spatial distribution of the deformation axes (fig. 2) shows a strong dispersion for the P-axes with a maximum in the $\mathrm{N} 40^{\circ}-60^{\circ} \mathrm{E}$ class. Conversely, the prevalence of the T-axes in the $\mathrm{N} 300^{\circ}-320^{\circ} \mathrm{E}$ is well evidenced. Both strike-slip and dip-slip solutions show deformation axes with dips generally less than $60^{\circ}$. No systematic variations of solutions with depth are observed.

The inversion of the whole dataset of focal mechanisms for stress tensor parameters gives an average misfit of $6.2^{\circ}$ that, on the basis of the error analysis, is near the value between acceptable and suspect results $\left(6^{\circ}\right)$ (Cocina et al., 1997). After dividing the entire dataset in two sub-volumes as a function of space, we found that the quality of the inversion was improved. In particular, the minimum compressive stress $\sigma_{3}$, estimated by stress inversion of the events located along the $\mathrm{CF}$ is horizontal and oriented $\mathrm{N} 145 \mathrm{E}$, while $\sigma_{l}$ is close to the vertical $\left(76^{\circ}\right.$ plunge). The inversion of the 37 remaining events, located westward and northwestward of $\mathrm{CF}$, again gives a horizontal $\sigma_{3}$, oriented approximately WNW-ESE, although with larger confidence regions.

The hypocentral distribution, the prevalence of focal mechanisms with fault planes striking about NE-SW, as well as the P- and T-axes distribution suggest that earthquakes occur along structures oriented ca. NE-SW, where surface evidence of this tectonic element is well recognized, and that the local stress field acting in this sector of the Southern Calabria is prevalently transtensional and strongly controlled by local fault heterogeneities. Interestingly, structural analysis, carried out by Tortorici et al. (1995) within the cataclastic belt developing along the $\mathrm{CF}$, show $\mathrm{N} 25-45^{\circ} \mathrm{E}$ trending minor fault planes characterized by subvertical slickensides (pitches ranging between $50^{\circ}$ and $90^{\circ}$ ), indicating $\mathrm{N} 140^{\circ} \mathrm{E}$ oriented extension (fig. 3d).

Analysis of focal mechanisms and seismogenic stress regimes carried out at regional scale in southern Italy (Frepoli and Amato, 2000a,b; Neri et al., 2003) and GPS results (Hollenstein et al., 2003; D'Agostino and Selvaggi, 2004) corroborate the hypothesis that the regional stress in the study area is dominated by a NW extension.

As concerns seismic attenuation, we found an average $Q_{P}=84 \pm 8$, which is lower than the $Q_{P}$ values observed in other tectonic areas (e.g., Giampiccolo et al., 2003). This suggests a high attenuation in southern Calabria which could be interpreted as due to a high degree of cracking and/or to the presence of fluids in the fractures. In addition, we observe some lateral variability of $Q_{P}$ among closely located stations. Figure 9 compares the $Q_{P}$ values along the source-to-receiver path obtained at each station with the information on $\mathrm{P}$-waves 3D velocity tomography performed in the region between 2 and $12 \mathrm{~km}$ of depth (Raffaele et al., 2006). In general, we observe a good agreement between both studies. In particular, high attenuation values (e.g. at stations DLV and CGT) well match with low $V_{P}$ volumes, whereas low attenuation (e.g. at station STC) is found in correspondence of high $V_{P}$ regions. Raffaele et al. (2006) interpreted the observed P-wave velocity anomalies in terms of geologic characteristics of the region. Following their interpretation, the observed P-wave anomalies may be attributed to the different percentages of major minerals in the rocks and to the respective single crystal velocities (Kern and Schenk, 1985). In fact, high contents of garnet, sillimanite, pyroxene and anphibole would produce high velocities and then low attenuation. On the other hand, material with low seismic velocities and high attenuation corresponds to rocks with low metamorphic grade. Moreover, the low $Q_{P}$ observed in the proximity of the CF (e.g. at station DLV), corresponding to negative $V_{P}$ anomaly, is in agreement with the interpretation of a zone of intense fracturing of fault gouge (Raffaele et al., 2006). This favours the hypothesis that the CF is a fault zone which gives rise to seismic activity.

Finally, source parameters of the earthquakes analysed are of the same order as those 


\section{Layer $z=2 \mathrm{~km}$}

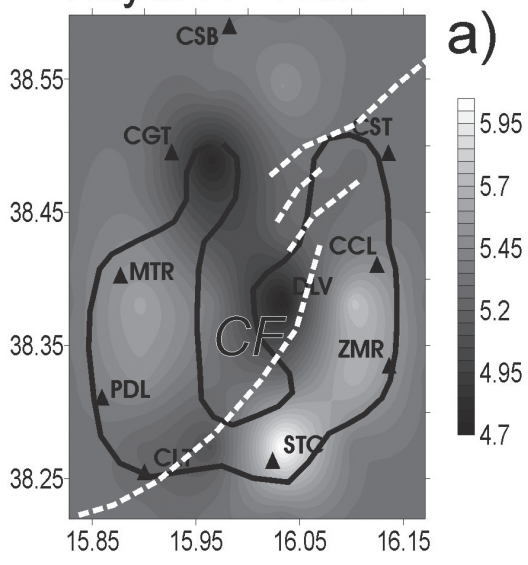

\section{Layer $z=9 \mathrm{~km}$}
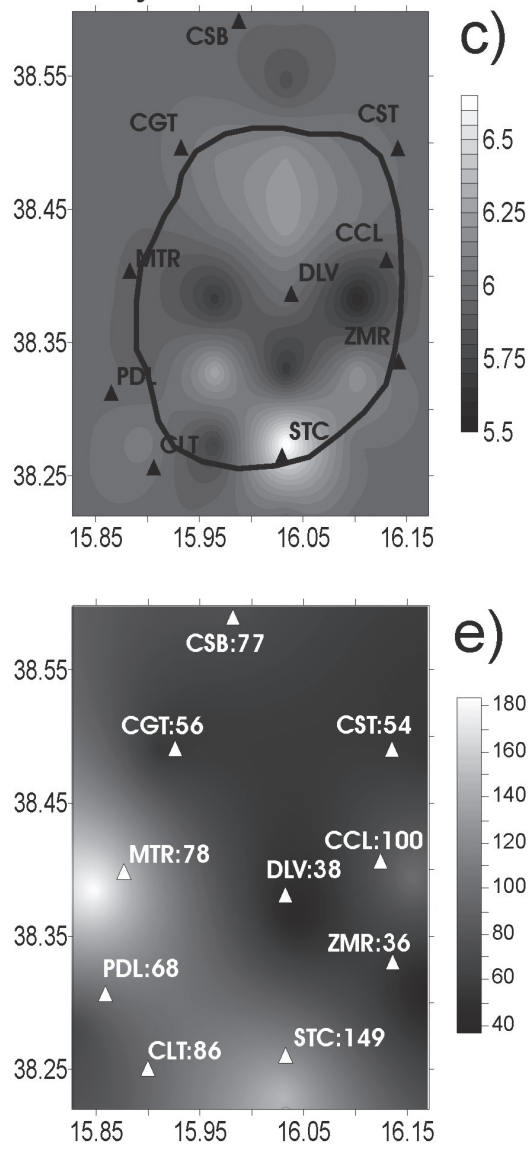

Layer $z=5 \mathrm{~km}$

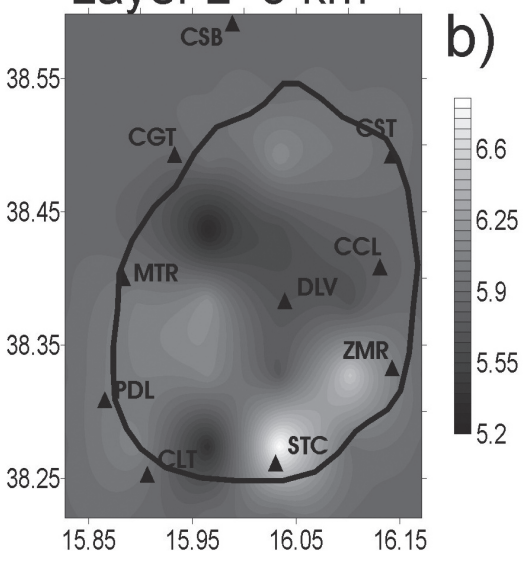

Layer $z=12 \mathrm{~km}$

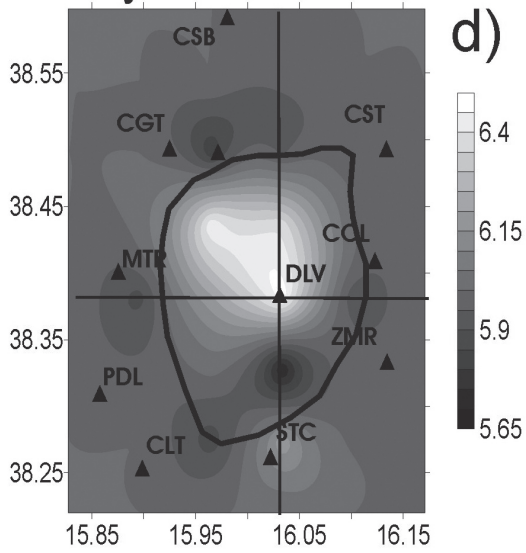

Fig. 9. Comparison between absolute P-wave velocity values (a-d) for the layers centred on 2, 5, 9 and $12 \mathrm{~km}$ depth (modified after Raffaele et al., 2006) and $Q_{P}$ values (e) obtained along the sourceto-receiver path at each station. The thick black contour denotes the limit of reliable resolution. In a structures are indicated with dotted white lines. 
reported in literature for other datasets of microearthquakes recorded in southern Calabria (e.g. Patanè et al., 1997) and in other tectonic settings (e.g., Abercrombie, 1995; Prejean and Ellsworth, 2001; Hough and Kanamori, 2002; de Lorenzo et al., 2004).

One interesting result regards the low stress drop estimates obtained for the selected earthquakes. In fact, most of the stress drop values are below 10 bars, except a few events that have stress drop up to 100 bars (fig.7a,b; table $\mathrm{V})$. Looking at the spatial distribution of stress drop (fig. 8), there is no evidence of any dependence on latitude, longitude and depth. Therefore, the general low values outline a characteristic of the whole region and are indicative of crustal heterogeneities, such as lowstrength structures (e.g. fault segments and/or weakened zones), where great stress accumulation is hindered. Since the rupture process on fault zones is heterogeneous, our findings are consistent with repeated ruptures of weak edge regions and, at the same time, do not exclude the presence of «strong» asperities were large stresses can be released (e.g. Sammis and Rice, 2001).

Computed source parameters allowed us to outline some scaling relationships for small earthquakes occurring in this tectonic area. From the obtained seismic moment-radius and seismic moment-stress drop relations it seems that, in the considered range of magnitude, the seismic moment increases with increasing source dimension and stress drop (fig. 7a,b). This observation implies a breakdown in the similarity of rupture processes for small earthquakes in the Gioia Tauro basin, as also observed by several authors worldwide (e.g. Izutani and Kanamori, 2001; de Lorenzo et al., 2004; Garcìa Garcìa et al., 2004), and favours the hypothesis that source processes of small earthquakes significantly differ from those of moderate to large events. On the other hand, plots of source dimension vs. seismic moment for dip-slip and strike-slip faults (the two faulting mechanisms mainly represented in the area) show the absence of any difference in the scaling behaviour (fig. 7c). Therefore, unlike moderate to large earthquakes, our results suggest that small earthquakes scale in the same man- ner, independently of faulting mechanism (e.g. Stock and Smith, 2001).

In conclusion, based on the findings of the present study we believe that the seismic activity in the study period reproduced fairly well the general features of seismicity in this specific area, even though other important seismogenic faults may have been inactive in such a relatively short period of time. In fact, seismogenic stress found to act over a 9-year time span is coherent with the main stress field inferred from geological data. This suggests that seismicity was produced by minor fault segments activated by the main stress field working in the whole region. However, to better constrain the seismotectonics of this high seismic risk area more data are needed and a more accurate knowledge of the crustal structure through high resolution investigations (e.g. velocity and attenuation tomographies) is required. To this end, continuing operation and technical improvement of existing earthquake monitoring networks (e.g. with a dense distribution of digital three-component seismic stations in key areas) should be planned in the future in order to improve the definition of specific structures and tectonic features.

\section{Acknowledgments}

We are grateful to ISMES for providing original data. We also thank Francesca Vella for her contribution in the data analysis and Giuseppe Di Grazia for helpful discussions. F.F. and S.G. have been supported by Catania University grants (Fondi di Ateneo-2005). Dr. R. Console, Prof. I. Guerra and two anonymous referees are gratefully acknowledged for useful comments and suggestions.

\section{REFERENCES}

ABERCROMBIE, R.E. (1995): Earthquake source scaling relationship from -1 to $5 \mathrm{M}_{\mathrm{L}}$ using seismograms recorded at 2.5-km depth, J. Geophys. Res., 100, 24.01524.036 .

BAKUn, W.H. and A. LINDTH (1977): Local magnitudes, seismic moments and coda durations for earthquakes near Oroville, California, Bull. Seism. Soc. Am., 65, 615-630. 
BOATWRIGHT, J. (1980): A spectral theory for circular seismic sources; simple estimates of source dimension, dynamic stress drop and radiated seismic energy, Bull. Seism. Soc. Am., 70, 1-27.

Boschi, E., E. Guidoboni, G. Ferrari, D. Mariotti and G. VALENSISE (2000): Catalogue of strong italian earthquakes, Ann. Geofis., 43, 268.

Cocina, O., G. Neri, E. Privitera and S. Spampinato (1997): Stress tensor computations in the Mount Etna area (southern Italy) and tectonic implications, J. Geodyn., 23, 109-127.

D'Agostino, N. and G. Selvaggi (2004): Crustal motion along the Eurasia-Nubia plate boundary in the Calabrian Arc and Sicily and active extension in the Messina Straits from GPS measurements, J. Geophys. Res., 109, B11402, doi:10.1029/2004JB002998.

De Lorenzo, S., G. Di Grazia, E. Giampiccolo, S. GresTA, H. LANGER, G. TuSA and A. URSino (2004): Source and Qp parameters from pulse width inversion of microearthquake data in southeastern Sicily, Italy, J. Geophys. Res., 109, B07308, doi:10.1029/2003JB002577.

Di Grazia, G., H. Langer, A. Ursino, L. Scarfì and S. GRESTA (2001): On the estimate of earthquake magnitude at a local seismic network, Ann. Geofis., 44, 579591.

FrePOLI, A. and A. AmATO (2000a): Fault plane solutions of crustal earthquakes in Southern Italy (1988-1995): seismotectonic implications, Ann. Geofis., 43, 437467.

Frepoli, A. and A. Amato (2000b): Spatial variation in stresses in peninsular Italy and Sicily from background seismicity, Tectonophysics, 317, 109-124.

Galli, P. and V. Bosi (2002): Paleoseismology along the Cittanova fault: Implications for seismotectonics and earthquake recurrence in Calabria (southern Italy), $J$. Geophys. Res., 92, 1029-1048.

Galli, P., and V. Bosi (2003): Catastrophic 1638 earthquakes in Calabria (southern Italy). New insight from paleoseismological investigation, J. Geophys. Res., 108, B1, doi:10.1029/2002JB0171.

Garcia Garcia J.M., M.D. Romacho and A. JimÉneZ (2004): Determination of near-surface attenuation, with $x$ parameter, to obtain the seismic moment, stress drop, source dimension and seismic energy for microearthquakes in the Granada Basin (Southern Spain), Phys. Earth Planet. Interiors, 141, 9-26.

GePhart, J.W. (1990): Stress and the direction of slip on fault planes, Tectonics., 9, 845-858.

GePharT, J.W. and D.W. ForsYTH (1984): An improved method for determining the regional stress tensor using earthquake focal mechanism data: application to the San Fernando earthquake sequence, J. Geophys. Res., 89, 9305-9320.

Giampiccolo, E., S. GResta and G. GANCi (2003): Attenuation of body waves in southeastern Sicily (Italy), Phys. Earth Planet. Inter., 135, 267-279.

Gillard, D., M: Wyss and P. OKubo (1996): Type of faulting and orientation of stress and strain as a function of space and time in Kilauea's south flank, Hawaii, $J$. Geophys. Res., 101, 16025-16042.

Gladwin, M.T. and F.D. STACEY (1974): Anelastic degradation of acoustic pulses in rock, Phys. Earth Planet. Interiors, 8 , 332-336.
Hollenstein, C., H.G. Kahle, A. Geiger, S. Jenny, S. Goes and D. Giardini (2003): New GPS constraints on the Africa-Eurasia plate boundary zone in southern Italy, Geophys. Res. Lett., 30, doi: 10.1029/2003GL 017554.

Hough, S.E. and H. Kanamori (2002): Source properties of earthquakes near the Salton Sea triggered by the 16 October 1999 M7.1 Hector Mine, California, earthquake, Bull. Seism. Soc. Am., 92, 1281-1289.

IZUTANI, Y. and H. KANAMORI (2001): Scale dependence of seismic energy-to-moment ratio for strike-slip earthquakes in Japan, Geophys. Res. Lett., 28, 4007-4010.

KeILIS-BoROK,V.I. (1959): On the estimation of the displacement in an earthquake source and of source dimension, Ann. Geofis., 12, 205-214.

KERN, H. and V. SchenK (1985): Elastic wave velocities in rocks from a lower crustal section in Southern Calabria (Italy), Phys. Earth Planet. Interiors, 40, 147-160.

KJaRTANSSON, E. (1979): Constant Q-wave propagation and attenuation, J. Geophys. Res., 84, 4737-4748.

LAHR, J.C. (1989): HYPOELLIPSE: A computer program for determining local earthquake hypocentral parameters, magnitude, and first motion pattern, USGS Open File Rept., 23/89, pp. 112.

MADARIAGA, R. (1976): Dynamics of an expanding circular fault, Bull. Seism. Soc. Am., 66, 639-666.

MichaEL, A.J. (1987): Use of focal mechanisms to determine stress: a control study, J. Geophys. Res., 92, 357368.

MoIA, F. (1987): Analisi sulla fenomenologia sismica del maggio 1985 avvenuta nello stretto di Messina ed osservata dalla rete sismica di Gioia Tauro, (VI Conf. G.N.G.T.S., Roma), 185-194 (in italian).

Monaco C. and L. TORTORICI (2000): Active faulting in the Calabrian Arc and eastern Sicily, J. Geodynamics, 29, 407-424.

Mulargia, F. and R.J. Geller (eds), (2003): Earthquake Science and Seismic Risk Reduction (Nato Science Series IV: Earth and Environmental Science-vol. 32), (Kluwer Academic Publisher).

Neri, G., G. Barberi, B. Orecchio and A. Mostaccio (2003): Seismic strain and seismogenic stress regime in the crust of the southern Thyrrenian region, Earth Planet. Sci. Lett., 213, 97-112.

PARKER, R.L. and M.K. McNuTT (1980): Statistics for the one norm misfit measure, J. Geophys. Res., 85, 44294430.

Patane, D., F. Ferrucci, E. Giampiccolo and L. ScaraMUZZINO (1997): Source scaling of microearthquakes at Mt. Etna volcano and in the Calabrian Arc (southern Italy), Geophys. Res. Lett., 24, 1879-1882.

Patane, D. and E. Privitera (2001): Seismicity related to 1989 and 1991-93 Mt. Etna (Italy) eruptions: kinematic constraints by fault solution analysis, J. Volcanol. Geotherm. Res., 109, 77-98.

Prejean S.G. and W. Ellsworth (2001): Observations of earthquakes source parameters at $2 \mathrm{~km}$ depth in the Long Valley Caldera, eastern California, Bull. Seism. Soc. Am., 91, 165-177.

Raffaele, R., H. Langer, S. Gresta and F. Moia (2006): Tomographic inversion of local earthquake data from the Gioia Tauro basin (southwestern Calabria, Italy), Geophys. J. Int., 165, 167-179. 
Reasenberg, P.A. and D. OpPenheimer (1985): Fortran computer programs for calculating and displaying earthquake fault-plane solutions, USGS Open File Rept., 379/85, pp. 109.

RiCHTER, C. (1935): An instrumental earthquake magnitude scale, Bull. Seism. Soc. Am., 25, 1-32.

Tortorici, L., C. Monaco, C. TAnsi and O. Cocina (1995): Recent and active tectonics in the Calabrian Arc (Southern Italy), Tectonophysics, 243, 37-55.

SAmmis, C.G. and J.R. RicE (2001): Repeating Earthquakes as Low-Stress-Drop Events at a Border between Locked and Creeping Fault Patches, Bull. Seism. Soc. Am., 91, 532-537.

Stock, C. and G.C. Euan Smith (2001): Evidence for different scaling of earthquake source parameters for large earthquakes depending on focal mechanism, Geophys J. Int., 143, 157-162.

VAlensise, G. and G. D'AdDEZIo (1994): Il contributo della geologia di superfice all'identificazione delle strutture sismogenetiche della Piana di Gioia Tauro,
Istituto Nazionale di Geofisica, Internal Report, 559, 21 (in italian).

Wallace, R.E. (1977): Profiles and ages of young fault scarps, north-central Nevada, Geol. Soc. Am. Bull., 88, 1267-1281.

Wu, H. and J.M. LEES (1996): Attenuation of Coso geothermal area, California, from wave pulse width, Bull. Seism. Soc. Am., 5, 1574-1590.

Wyss, M., B. Liang, W.R. Tanigawa and W. Xiaoping (1992): Comparison of orientations of stress and strain tensor based on fault plane solutions in Kaoiki, Hawaii, J. Geophys. Res., 97, 4769-4790.

Zollo, A. and S. DE LOREnZo (2001): Source parameters and three-dimensional attenuation structure from the inversion of microearthquake pulse width data: Method and synthetic tests, J. Geophys. Res., 106, 1628716306.

(received March 27, 2008; accepted September 18, 2008) 